\title{
Effectiveness of Biofeedback Therapy in Patients with Bowel Dysfunction Following Rectal Cancer Surgery: A Systemic Review with Meta-Analysis
}

\author{
Haoze Li (D), Ce Guo, Jiale Gao, Hongwei Yao \\ Department of General Surgery, Beijing Friendship Hospital, Capital Medical University, Beijing Key Laboratory of Cancer Invasion and Metastasis \\ Research and National Clinical Research Center for Digestive Diseases, Beijing, 100050, People's Republic of China \\ Correspondence: Hongwei Yao, Department of General Surgery, Beijing Friendship Hospital, Capital Medical University, Beijing Key Laboratory of \\ Cancer Invasion and Metastasis Research and National Clinical Research Center for Digestive Diseases, 95 Yong-an Road, Xi-Cheng District, Beijing, \\ 100050, People's Republic of China, Email yaohongwei@ccmu.edu.cn
}

Objective: To identify, systematically review and synthesize the evidence on the effectiveness of biofeedback therapy in patients with bowel dysfunction following rectal cancer surgery.

Data Sources: Four electronic databases (PubMed 1974-2021; Embase1980-2021; Cochrane databases and the trial registers) were systematically searched by reviewers from inception through March 2021.

Study Selection: Randomized controlled trials (RCTs), cohort studies, and case series studies were included for adults with bowel dysfunction following rectal cancer surgery. All participants received an intervention of biofeedback treatment. Any outcomes that can evaluate the patient's bowel function were the primary research endpoint, while the quality of life was the second endpoint. The disagreements between the two reviewers were resolved after discussion and the third independent reviewer's ruling. As a result, 12 of 185 studies met selection criteria and were included in the review.

Data Extraction: We designed an electronic data extraction form and data were extracted independently. The methodological quality of included studies was assessed using the Cochrane Risk of Bias, the MINORS scale, and the Institute of Health Economics scale. Data Synthesis: Meta-analyses were conducted for case series only and narrative syntheses were completed. Key findings included significant improvements in bowel function as well as health-related quality of life after biofeedback therapy. (Wexner score: $\mathrm{t}=7$, $\mathrm{MD}=3.33$; $95 \%$ CI $[2.48,4.18]$ ) and (Vaizey score: $\mathrm{t}=3, \mathrm{MD}=2.46$; $95 \%$ CI $[1.98,2.93])$. Subgroup analysis of Wexner score: receiving electrical stimulation therapy $(\mathrm{t}=3, \mathrm{MD}=2.36 ; 95 \% \mathrm{CI}[1.51,3.22])$, not receiving electrical stimulation $(\mathrm{t}=4, \mathrm{MD}=3.79 ; 95 \%$ $\mathrm{CI}[2.66,4.93])$; not receiving adjuvant chemoradiotherapy $(\mathrm{t}=3, \mathrm{MD}=2.42 ; 95 \% \mathrm{CI}[1.61,3.24])$, chemotherapy and radiotherapy $(\mathrm{t}=1$, $\mathrm{MD}=4.10 ; 95 \% \mathrm{CI}[2.90,5.30])$, radiotherapy and chemotherapy on parts of patients $(\mathrm{t}=2, \mathrm{MD}=3.46 ; 95 \% \mathrm{CI}[1.41,5.51])$, chemotherapy ( $\mathrm{t}=1, \mathrm{MD}=4.81 ; 95 \% \mathrm{CI}[3.38,6.24])$; performing ISR ( $\mathrm{t}=2, \mathrm{MD}=3.32 ; 95 \%$ CI $[0.37,6.27])$, performing AR ( $\mathrm{t}=4$, $\mathrm{MD}=3.08 ; 95 \% \mathrm{CI}[2.12,4.04])$, performing PLRAS surgery $(\mathrm{t}=1, \mathrm{MD}=4.10 ; 95 \% \mathrm{CI}[2.90,5.30])$.

Conclusion: Although biofeedback therapy may improve intestinal function and quality of life as well as anal function reflected by ARM after surgery, patient satisfaction is still unclear. Due to the scarcity of data, good-quality research is required to delve deeper. Clinical Trial Registration Number: CRD42020192658.

Keywords: biofeedback, fecal incontinence, rectal neoplasms, surgery, rehabilitation

\section{Introduction}

Colorectal cancer has the third incidence $(10 \%)$ and the second mortality $(9.4 \%)$ of malignant tumors in the world. ${ }^{1}$ Nevertheless, benefiting from the continuous maturity of adjuvant chemoradiotherapy and the continuous improvement of surgical technology, the 5-year survival rate of colorectal cancer has increased from $50 \%$ in the mid-1970s to $64 \%$ in 2009-2015. ${ }^{2}$ Surgical treatment, including sphincter-preserving operations, is the preferred treatment for rectal cancer. ${ }^{3,4}$ After rectal cancer surgery, various intestinal dysfunctions occur, primarily manifested by defecation disorders resulting from changes in the rectal structure, tissue injuries such as sphincters and nerves, and defecation reflex decline. ${ }^{5}$ 
Therefore, functional rehabilitation training for patients after rectal cancer surgery has become worthy of our attention. Biofeedback therapy is increasingly being used as a rehabilitation tool to help patients improve their intestinal function. ${ }^{6,7}$

Biofeedback is a training method that aims to improve the conscious control of the individual's involuntary physiological activity to improve his/her physical, emotional, mental, and spiritual health. ${ }^{8}$ It can show patients how the anal sphincter works via visual or verbal feedback through the monitor and teach them to distinguish anal sphincter contraction from the buttock and abdominal muscle effort. Aiming to improve postoperative intestinal function in patients with rectal cancer, patients received sensory, strength, and coordination training for anal sphincter, pelvic floor muscle, and puborectalis muscle as biofeedback treatment. ${ }^{9}$ The advantages of biofeedback therapy can provide opportunities to improve the accuracy of functional tasks, increase the participation of patients in rehabilitation, and reduce the need for ongoing contact with healthcare professionals to monitor the implementation of rehabilitation programs. ${ }^{10}$

Biofeedback therapy is not routinely used in the rehabilitation training of patients with intestinal dysfunction after rectal cancer surgery, and there is no national recognition or professional guidance for this therapy. However, a strong interest has been aroused in biofeedback therapy in rehabilitation medicine and colorectal surgery, and biofeedback technology is constantly developing. Few systematic reviews or meta-analyses have reported on the impact of biofeedback therapy on patients with bowel dysfunction after surgery. Moreover, problems such as insufficient sample size and fewer RCT studies exist in the published reviews. So we conducted a systematic review and meta-analysis to describe the evidence for the effectiveness of biofeedback therapy in patients with bowel dysfunction following rectal cancer surgery.

\section{Methods}

This review is accomplished strictly under the Preferred Reporting Items for Systematic Reviews and Meta-Analyses (PRISMA) Guide. ${ }^{11,12}$ The review protocol has been registered in advance (PROSPERO CRD42020192658). According to the search habits of different databases, the following terms were used comprehensively by reviewers (GJL) to explore the literature in PubMed (1974-2021), Embase (1980-2021), the Cochrane database, and the trial registers: Fecal Incontinence; Bowel Incontinence; Fecal Soiling; Low anterior resection syndrome; resection syndrome; Bowel dysfunction; Bowel function; Sphincter function; intestinal dysfunction; intestinal function; biofeedback; feedback; Rectal Neoplasms; Rectal Tumors; Rectum Cancer (Please refer to Appendix 1 for the specific search strategy and search results). None of the articles were excluded because of language or publication time. The last retrieval time was May 13, 2021. Supplementary File 1 displays the details.

\section{Study Selection}

Two reviewers (LHZ and GC) respectively carried out literature screening of all retrieved articles. The disagreements were resolved after discussion and the third independent reviewer's ruling. As a result, the following inclusion criteria were formulated: 1. Participants: Patients of any age suffering from malignant rectum tumors (Patients with benign rectal disease and colon disease were excluded); the study included patients undergoing surgical treatment (regardless of the surgical type and whether preoperative neoadjuvant therapy or postoperative radiotherapy and chemotherapy were implemented); 2. Intervention: Biofeedback treatment with or without PFMT (Pelvic Floor Muscle Training) or electrical stimulation treatment $t$ (the type, frequency, and duration of biofeedback treatment were not limited); 3 . Outcomes: Primary research endpoint (any questionnaire that can evaluate the patient's bowel function, patient feedback, and intestinal diary, anal manometry, ultrasonic endoscope, etc.); secondary study endpoint (Quality of life after biofeedback treatment); 4. Types of studies: RCTs (randomized controlled trials), cohort studies, case series (not including case studies with only one participant), because the data can fully reflect the effectiveness of biofeedback therapy in patients with bowel dysfunction following rectal cancer surgery. Studies were excluded if they were non-clinical studies or letters to the editor or expert consensus. 


\section{The Process of Data Extraction}

An electronic data extraction form was specially designed to reflect the data extraction process and ensure the accuracy of the extracted data. Two reviewers (LHZ and GC) carried out data extraction separately and double-checked the accuracy of the data. The disagreements were resolved after discussion. The electronic data extraction form contains the following items: 1. Study summary (Review Title, Date, Reviewer, Study Title, First author, Year of publication, Country of publication, publication type, author contact information); 2. The included article characteristics (research type, whether the evaluation meets the inclusion criteria, the number of participants, gender, age, intervention group, and control group measures, follow-up time, description of outcome indicators); 3 . The included study results (Please refer to Appendix 2 for electronic data extraction form). ${ }^{13}$

\section{Risk of Bias}

RCT studies, cohort studies, and case series studies were included in this research. We assessed the risk of bias in RCT studies using the Cochrane quality assessment tool, ${ }^{14}$ in cohort studies using the MINORS scale,${ }^{15}$ in case series using the Institute of Health Economics scale for Case Series. ${ }^{16}$ The risk bias assessment of literature was reviewed by two team members independently. Disagreements were resolved through discussion, resulting in unified results.

\section{Analysis}

We carried out the quantitative analysis and meta-analysis based on the literature whose outcome indicators were complete and adequate. Systematic reviews were carried out on the remaining portion of the literature. Since most of the literature available for meta-analysis is case series studies with continuous variables, most outcomes are presented by mean. The review was conducted using StataMP16 (64- bit) for meta-analysis. We used the fixed effect model and the random effect model to combine effect quantities. Heterogeneity is quantified mainly by the value of $\mathrm{I}^{2}$. If heterogeneity is $\left(\mathrm{I}^{2}>50 \%\right)$, a random effect model is used to calculate the results. Conversely, if heterogeneity is $\left(\mathrm{I}^{2} \leq 50 \%\right)$, a fixed effect model is used to calculate the results. ${ }^{17}$ For further discussion, heterogeneity was assessed in the process of metaanalysis. A subgroup analysis was conducted to examine the surgery type, electrical stimulation, and adjuvant therapy. Data has been presented in the form of images in this regard. We used an online tool to extract data from images. (https:// apps.automeris.io/wpd/index.zh_CN.html). We contacted the article's author via email and requested the original data (2 replies were received with no other data, and 1 did not reply out of 3 emails). Due to the limited number of studies, no funnel plot was proposed. ${ }^{18}$

\section{Results}

\section{Study Selection}

A total of 285 articles were retrieved after searching the electronic database mentioned earlier. 252 articles were excluded after removing duplicate articles and reading titles or abstracts roughly. 17 articles did not meet the inclusion criteria (Please refer to Appendix 3): not rectal disease (including colon disease or other diseases): 6; No surgery performed: 5; rectal benign tumor surgery (including endoscopic surgery, Transanal minimally Invasive surgery, Transanal Endoscopic Microsurgery, etc.): 4; containing the same data: 2. After re-reading the full text and eliminating four articles with no clinical data, a total of 12 articles with extractable and analytic data were included. Figure 1 displays the detailed study selection process. ${ }^{18-30}$

\section{Study Characteristics}

The review includes 12 studies ( 2 RCTs; $;{ }^{28,30} 2$ no randomized controlled trials; ${ }^{25,26} 8$ case series ${ }^{18-24,27,29}$ ).A total of 561 patients who suffered from bowel dysfunction after rectal cancer were included in this review, of whom 422 patients received biofeedback treatment as the intervention group, 103 patients did not receive any intervention, and 36 patients received PFMT only without biofeedback as a controlled group. 


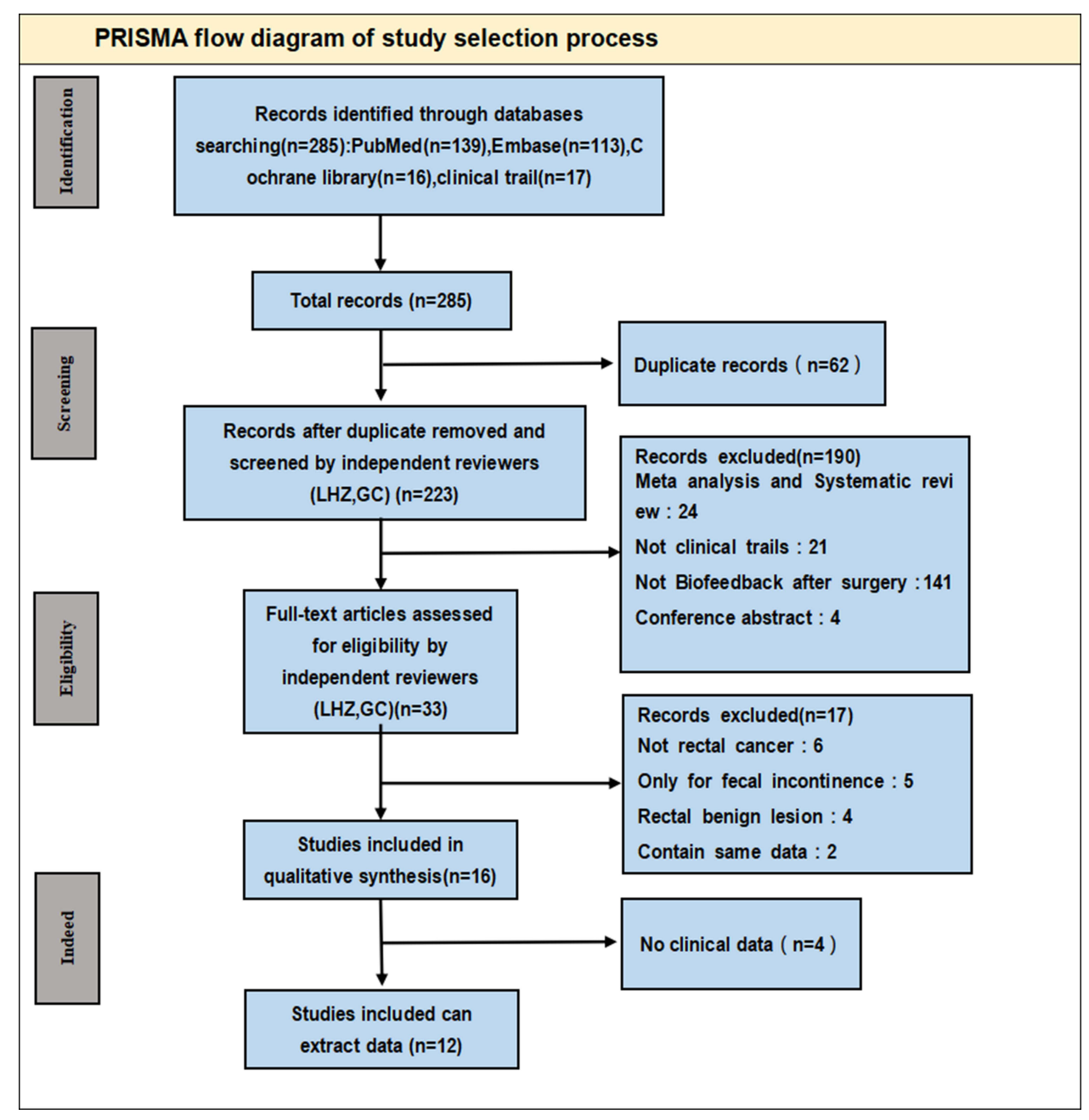

Figure I PRISMA flow diagram of study selection process.

\section{Participants' Characteristics}

In both the intervention and control groups, male patients accounted for a higher or roughly equal proportion of female patients, while only two studies found female patients significantly outnumbered male patients. ${ }^{20,23}$ It may be related to the higher incidence of rectal cancer in male patients than in female patients. ${ }^{1}$ The majority of patients in all 12 studies were older, middle-aged, and younger elderly people, with a range of mean (SD) ages ranging from 51.2-67.6; 3 studies $^{25,28,29}$ did not mention the approach of surgery specifically (just mentioned the anastomotic method or sphinctersaving TME); 1 study implemented $A R ;{ }^{24} 3$ studies implemented LAR; ${ }^{19,21,30} 2$ studies implemented ISR; ${ }^{20,27} 2$ studies implemented different procedures for different patients (AR, LAR, ULAR, ISR); ${ }^{22,26}$ Table 1 displays the detailed participants' characteristics.

\section{Intervention Characteristics}

Although all included studies operated biofeedback therapy on patients with intestinal dysfunction after resection of rectal cancer, different studies had different measures. From surgery to biofeedback treatment, the time ranges from 1 month, half a year, and 1 year. The duration of biofeedback treatment ranges from 20 to 90 minutes, while the frequency ranges from 1 to 3 weeks per time. Some studies performed enhanced-frequency (6 to 32 weeks) ${ }^{21}$ biofeedback treatments to improve efficacy. In most studies, PEF or electrical stimulation was performed together with biofeedback treatment, and visual or visual + verbal feedback was used for the strength, sensation, and coordination training for anal muscles. Table 2 displays the detailed Intervention and outcome characteristics. 
Table I Participants Characteristics in Included Studies

\begin{tabular}{|c|c|c|c|c|c|c|c|}
\hline Author and Year & Sex,(M/F) & Age(Years) & Surgery Type & Tumor Height (cm) & $\begin{array}{l}\text { Anostomosis } \\
\text { Height }(\mathrm{cm})\end{array}$ & Adjuvant Therapy & Ostomy \\
\hline \multicolumn{8}{|l|}{ Prospective randomized trials } \\
\hline $\begin{array}{l}\text { Kye et al } 2016^{28} \\
N=47 \\
\text { (BFT Group:21; Control Group:26) }\end{array}$ & $\begin{array}{c}\text { BFT Group } \\
\text { M:10(47.6\%) F: I I (52.4\%) } \\
\text { Control Group(Conservative Self- } \\
\text { rehabilitation) } \\
\text { M:15 (57.7\%) F:15 (57.7\%) }\end{array}$ & $\begin{array}{l}\text { BFT Group } \\
61.7 \pm 9.8^{\ddagger} \\
\text { Control Group } \\
64.5 \pm 9.4^{\ddagger}\end{array}$ & $\begin{array}{l}\text { BFT Group } \\
\text { CRA:15 (71.4\%) } \\
\text { CAA:6 (28.6\%) } \\
\text { Control Group } \\
\text { CRA:23 (88.5\%) } \\
\text { CAA:3 (11.5\%) }\end{array}$ & $\begin{array}{c}\text { BFT Group } \\
\leq 5 \mathrm{~cm}: 6(28.6 \%) \\
>5 \mathrm{~cm}: 15(71.4 \%) \\
\text { Control Group } \\
\leq 5 \mathrm{~cm}: 8(30.8 \%) \\
>5 \mathrm{~cm}: 18(69.2 \%)\end{array}$ & NR & $\begin{array}{l}\text { BFT Group } \\
\text { Pre-op:CRT } \\
\text { Control Group } \\
\text { Post-op:CT }\end{array}$ & All \\
\hline $\begin{array}{l}\text { Zheng et al } 2019^{30} \\
\mathrm{~N}=109 \\
\text { (Blank control group:38; PFMT Group:36; } \\
\text { BFT Group:35) }\end{array}$ & $\begin{array}{c}\text { Blank control group M:30(79\%) F:8 } \\
\text { (21\%) } \\
\text { PFMT Group } \\
\text { M:27 (75\%) F:9 (25\%) } \\
\text { BFT Group } \\
\text { M:25 (71\%) F:10 (29\%) }\end{array}$ & $\begin{array}{c}\text { Blank control group } \\
51.2 \pm 12.3^{\ddagger} \\
\text { PFMT Group } \\
52.5 \pm 10.4^{\ddagger} \\
\text { BFT Group } \\
54.3 \pm 9.9^{\ddagger}\end{array}$ & Dixon & $\begin{array}{c}\text { Blank control group } \\
5.1 \pm 1.9^{\ddagger} \\
\text { PFMT Group } \\
5.1 \pm 1.9^{\ddagger} \\
\text { BFT Group } \\
5.0 \pm 1.6^{\ddagger}\end{array}$ & NR & Pre-op:CRT & All \\
\hline \multicolumn{8}{|l|}{ Prospective non-randomized trials } \\
\hline $\begin{array}{l}\text { Laforest et al } 2012^{25} \\
\mathrm{~N}=46 \\
\text { (Rehabilitation Group:22; Control } \\
\text { Group:24) }\end{array}$ & $\begin{array}{l}\text { Rehabilitation Group } \\
\text { M:I I (50.0\%) F: I I (50.0\%) } \\
\quad \text { Control Group } \\
\text { M:I5 (62.5\%) F:9 (37.5\%) }\end{array}$ & $\begin{array}{l}\text { Rehabilitation } \\
\text { Group: } 55 \\
\text { (33-78)* } \\
\text { Control Group: } \\
\text { 60(35-80)* }\end{array}$ & $\begin{array}{l}\text { Laparoscopic } \\
\text { sphincter-saving } \\
\text { TME }\end{array}$ & $\begin{array}{l}\text { Rehabilitation Group:3 } \\
\text { (0.5-9.0)* } \\
\text { Control Group:2.5(I.0- } \\
\text { 9.0)* }\end{array}$ & $\begin{array}{l}\text { Rehabilitation Group: } \\
0.5(0-3.0)^{*} \\
\text { Control Group:NR }\end{array}$ & $\begin{array}{c}\text { Pre-op CRT } \\
32 \text { (69.6\%) }\end{array}$ & NR \\
\hline $\begin{array}{l}\text { Lee et al } 2019^{26} \\
\mathrm{~N}=31 \\
\text { (BFT Group:16;Control Group:15) }\end{array}$ & $\begin{array}{c}\text { BFT Group } \\
\text { M:7(43.8\%) F:9(56.2\%) } \\
\text { Control Group } \\
\text { M:12 (80.0\%) F:3(20.0\%) }\end{array}$ & $\begin{array}{c}\text { BFT Group } \\
<70: 14(87.5 \%) \\
\geq 70: 2(12.5 \%) \\
\text { Control Group } \\
<70: 9(60.0 \%) \\
\geq 70: 6(40.0 \%)\end{array}$ & $\begin{array}{c}\text { BFT Group } \\
\text { Low anterior resection:13 } \\
(81.2 \%) \\
\text { Intersphincteric } \\
\text { resection:3(18.8\%) } \\
\text { Control Group } \\
\text { Low anterior resection: } 14 \\
(93.3 \%) \\
\text { Intersphincteric } \\
\text { resection:I }(6.7 \%)\end{array}$ & $\begin{array}{c}\text { BFT Group } \\
<5 \mathrm{~cm}: 8(50.0 \%) \\
\geq 5 \mathrm{~cm}: 8(50.0 \%) \\
\text { Control Group } \\
<5 \mathrm{~cm}: 1(6.7 \%) \\
\geq 5 \mathrm{~cm}: 14(93.3 \%)\end{array}$ & $\begin{array}{c}\text { BFT Group } \\
<5 \mathrm{~cm}: 13(81.2 \%) \\
\geq 5 \mathrm{~cm}: 3(18.8 \%) \\
\text { Control Group } \\
<5 \mathrm{~cm}: 5(33.3 \%) \\
\geq 5 \mathrm{~cm}: 10(66.7 \%)\end{array}$ & Pre-op:RT 6 (19.4\%) & $\begin{array}{c}12 \\
(38.7 \%)\end{array}$ \\
\hline \multicolumn{8}{|l|}{ Case series } \\
\hline $\begin{array}{l}\text { Allgayer et al } 2005^{19} \\
\mathrm{~N}=95 \text { (Irradiated patients:41;Non- } \\
\text { irradiated patients:54) }\end{array}$ & $\begin{array}{c}\text { Irradiated M:28(68.3\%)F:13(31.7\%) } \\
\text { Non-irradiated } \\
\text { M:33(61.1\%)F:(38.9\%) }\end{array}$ & $\begin{array}{l}\text { Irradiated: } 58.5(31- \\
\text { 76)* } \\
\text { Non-irradiated: } 67 \\
\quad(48-83)^{*}\end{array}$ & LAR & NR & $\begin{array}{c}\text { Irradiated: } \\
7.6 \pm 3.1^{\ddagger} \\
\text { Non-irradiated: } 10.3 \\
\pm 4.2^{\ddagger}\end{array}$ & Post-op: RT 4 I (43.2\%) & NR \\
\hline
\end{tabular}


Table I (Continued).

\begin{tabular}{|c|c|c|c|c|c|c|c|}
\hline Author and Year & Sex,(M/F) & Age(Years) & Surgery Type & Tumor Height $(\mathrm{cm})$ & $\begin{array}{l}\text { Anostomosis } \\
\text { Height }(\mathrm{cm})\end{array}$ & Adjuvant Therapy & Ostomy \\
\hline $\begin{array}{l}\text { Cong et al } 2006^{20} \\
N=16\end{array}$ & M:5 F:II & $56(4 I-74)$ & ISR & $3.3(2-4.5)^{*}$ & NR & NR & NR \\
\hline $\begin{array}{l}\text { Du et al } 2010^{21} \\
N=24\end{array}$ & M:16 F:8 & $67.6 \pm 10.5^{\ddagger}$ & LAR & $6.6 \pm 3.6^{\ddagger}$ & NR & No & NR \\
\hline $\begin{array}{l}\text { Bartlett et al } 2011^{22} \\
N=19\end{array}$ & M:10 F:9 & $\begin{array}{c}64.1 \text { (95\% Cl: } 47.0- \\
81.3) \dagger\end{array}$ & $\begin{array}{c}\text { Anterior resection: } 3 \\
(15.8) \% \\
\text { Ultra-low anterior } \\
\text { Resection: } 10 \\
(52.6 \%) \\
\text { Segmental colectomy:2 } \\
(10.5 \%) \\
\text { Proctocolectomy } \\
4(21.1 \%)\end{array}$ & NR & NR & NR & NR \\
\hline $\begin{array}{l}\text { Cong et al } 2011^{23} \\
N=11\end{array}$ & M:4 F:7 & $51(38-65)^{*}$ & PLRAS & NR & NR & Pre-op:CRT & All \\
\hline $\begin{array}{l}\text { Kim et al } 201 I^{24} \\
N=70\end{array}$ & M:49 F:2I & $58.1 \pm 10.1^{\ddagger}$ & $A R$ & NR & $4.1 \pm 1.8^{\ddagger}$ & $\begin{array}{c}\text { Pre-op:CT:I(I.4\%) } \\
\text { RT:30(42.9\%) } \\
\text { Post-op: CT:25(35.7\%) } \\
\text { RT:19(27.1\%) } \\
\text { Both: } \\
\text { CT:3I }(44.3 \%)\end{array}$ & $21(30 \%)$ \\
\hline $\begin{array}{l}\text { Kuo et al } 2015^{27} \\
N=32\end{array}$ & M:17 F:I5 & $56.5(3 \mathrm{I}-70)^{*}$ & ISR & $3.89(1.5-5.0)^{*}$ & NR & Pre-op:CRT25 (78.1\%) & $\begin{array}{c}5 \\
(15.6 \%)\end{array}$ \\
\hline $\begin{array}{l}\text { Liang et al } 2016^{29} \\
N=61\end{array}$ & $M: 40 F: 21$ & $63.1 \pm 10.5^{\ddagger}$ & TME & NR & $5.2 \pm 3.1^{\ddagger}$ & $\begin{array}{l}\text { RT:I4(23.0\%) } \\
\text { CT:13(21.3\%) }\end{array}$ & No \\
\hline
\end{tabular}

Notes: Values in parentheses are percentages unless indicated otherwise; values are median (range)*, mean $\left(95 \% \mathrm{Cl}\right.$.) † Mean \pm SD ${ }^{\ddagger}$.

Abbreviations: M, male; F, female; BFT, Biofeedback therapy; CRA, Colorectal anastomosis; CAA, Coloanal anastomosis; NR, not reported; Pre-op, Pre-operation; Post-op, Post-operation; CT, Chemotherapy; RT, Radiotherapy; CRT, Chemoradiotherapy; PFMT, Pelvic Floor Muscle Training; TME, Total Mesorectal Excision; LAR, Low Anterior Resection; ISR, Intersphincteric Resection. 
Table 2 Intervention and outcomes in Included Studies

\begin{tabular}{|c|c|c|c|c|c|c|c|c|c|}
\hline Author and Year & $\begin{array}{l}\text { Time from Surgery to } \\
\text { Biofeedback (Week) }\end{array}$ & $\begin{array}{l}\text { Training } \\
\text { Type }\end{array}$ & $\begin{array}{l}\text { BF } \\
\text { Type }\end{array}$ & $\begin{array}{l}\text { Practice } \\
\text { Type at } \\
\text { Home }\end{array}$ & $\begin{array}{c}\text { BFT } \\
\text { Duration } \\
\text { (min) }\end{array}$ & Frequency & $\begin{array}{l}\text { Length } \\
\text { (Week) }\end{array}$ & $\begin{array}{l}\text { Outcomes (Bowel } \\
\text { Function) }\end{array}$ & $\begin{array}{l}\text { Outcomes } \\
\text { (Quality of } \\
\text { Life) }\end{array}$ \\
\hline \multicolumn{10}{|l|}{ Prospective randomized trials } \\
\hline $\begin{array}{l}\text { Kye et al } 2016^{28} \\
\mathrm{~N}=47 \\
\text { (BFT Group:21; Control } \\
\text { Group:26) }\end{array}$ & During stoma interval & $N R$ & NR & PEFT & NR & I-2/week & 10 & $\begin{array}{c}\text { ARM } \\
\text { Number of daily } \\
\text { defecation } \\
\text { CCIS score }\end{array}$ & NR \\
\hline $\begin{array}{l}\text { Zheng et al } 2019^{30} \\
\text { N=109 } \\
\text { (Blank control group:38; } \\
\text { PFMT Group:36; } \\
\text { BFT Group:35) }\end{array}$ & 64 & $\begin{array}{l}\text { Strength } \\
\text { training }\end{array}$ & $\begin{array}{l}\text { Visual } \\
\text { Verbal }\end{array}$ & PEFT & 20 & $3 /$ week & 16 & MSKCC & NR \\
\hline \multicolumn{10}{|c|}{ Prospective non-randomized trials } \\
\hline $\begin{array}{l}\text { Laforest et al } 2012^{25} \\
\mathrm{~N}=46 \\
\text { (Rehabilitation Group:22; } \\
\text { Control Group:24) }\end{array}$ & NR & NR & Visual & PEFT & 60 & I/week & 15 & $\begin{array}{l}\text { Gastrointestinal } \\
\text { standardized } \\
\text { questionnaire } \\
\text { Wexner score } \\
\text { Kirwan's classification }\end{array}$ & $\begin{array}{l}\text { SF-36 } \\
\text { FIQL }\end{array}$ \\
\hline $\begin{array}{l}\text { Lee et al } 2019^{26} \\
\mathrm{~N}=31 \\
\text { (BFT Group: 16;Control } \\
\text { Group:15) }\end{array}$ & NR & NR & Visual & PEFT & NR & $2 /$ week & 5 & $\begin{array}{c}\text { ARM } \\
\text { Wexner score NBM } \\
\text { LARS }\end{array}$ & NR \\
\hline \multicolumn{10}{|l|}{ Case series } \\
\hline $\begin{array}{l}\text { Allgayer et al } 2005^{19} \\
\text { N=95(Irradiated patients: } 41 ; \\
\text { Non-irradiated patients:54) }\end{array}$ & $6(4-40)^{*}$ & $\begin{array}{l}\text { Strength } \\
\text { training }\end{array}$ & Visual & PEFT & 60 & Daily & 3 & $\begin{array}{l}\text { ARM } \\
\text { MCIS } \\
\text { EUS }\end{array}$ & NR \\
\hline $\begin{array}{l}\text { Cong et al } 2006^{20} \\
N=16\end{array}$ & 4 & $\begin{array}{l}\text { Strength } \\
\text { training } \\
\text { Sensory } \\
\text { training } \\
\text { Coordination } \\
\text { training }\end{array}$ & $\begin{array}{l}\text { Visual } \\
\text { Verbal }\end{array}$ & $\begin{array}{c}\text { PFET } \\
\text { Electrical } \\
\text { stimulation }\end{array}$ & $\begin{array}{c}\text { PEF:30 } \\
\text { Electrical } \\
\text { stimulation: } 15\end{array}$ & $\begin{array}{l}\text { Strength training:I/week Sensory } \\
\text { training:2/week Electrical stimulation:I/ } \\
\text { week }\end{array}$ & 3 & $\begin{array}{c}\text { ARM } \\
\text { Vaizey score } \\
\text { Wexner score }\end{array}$ & NR \\
\hline
\end{tabular}


Table 2 (Continued)

\begin{tabular}{|c|c|c|c|c|c|c|c|c|c|}
\hline Author and Year & $\begin{array}{l}\text { Time from Surgery to } \\
\text { Biofeedback (Week) }\end{array}$ & $\begin{array}{l}\text { Training } \\
\text { Type }\end{array}$ & $\begin{array}{l}\text { BF } \\
\text { Type }\end{array}$ & $\begin{array}{l}\text { Practice } \\
\text { Type at } \\
\text { Home }\end{array}$ & $\begin{array}{c}\text { BFT } \\
\text { Duration } \\
\text { (min) }\end{array}$ & Frequency & $\begin{array}{l}\text { Length } \\
\text { (Week) }\end{array}$ & $\begin{array}{l}\text { Outcomes (Bowel } \\
\text { Function) }\end{array}$ & $\begin{array}{l}\text { Outcomes } \\
\text { (Quality of } \\
\text { Life) }\end{array}$ \\
\hline $\begin{array}{l}\text { Du et al } 2010^{21} \\
N=24\end{array}$ & NR & $\begin{array}{l}\text { Strength } \\
\text { training } \\
\text { Sensory } \\
\text { training } \\
\text { Coordination } \\
\text { training }\end{array}$ & Visual & $\begin{array}{c}\text { PFET } \\
\text { Electrical } \\
\text { stimulation }\end{array}$ & $\begin{array}{c}\text { PEF:30 } \\
\text { Electrical } \\
\text { stimulation:15 }\end{array}$ & PEF:I-2/day electrical Stimulation:I/day & 6-32 & $\begin{array}{c}\text { ARM } \\
\text { Vaizey score } \\
\text { Wexner score }\end{array}$ & NR \\
\hline $\begin{array}{l}\text { Bartlett et al } 2011^{22} \\
N=19\end{array}$ & $18(12-24)^{*}$ & $\begin{array}{c}\text { Strength } \\
\text { training } \\
\text { Sensory } \\
\text { training } \\
\text { Coordination } \\
\text { training }\end{array}$ & $\begin{array}{l}\text { Visual } \\
\text { Verbal }\end{array}$ & $\begin{array}{c}\text { Relaxation } \\
\text { breathing } \\
\text { Anal squeeze } \\
\text { PEFT } \\
\text { Evacuation } \\
\text { techniques }\end{array}$ & $60-90$ & Daily & mean: 7 & $\begin{array}{l}\text { ARM The Continence } \\
\text { Grading Scale VAS }\end{array}$ & FIQL \\
\hline $\begin{array}{l}\text { Cong et al } 2011^{23} \\
\mathrm{~N}=11\end{array}$ & NR & NR & NR & NR & NR & NR & NR & $\begin{array}{l}\text { Vector manometry } \\
\text { Wexner score }\end{array}$ & $\begin{array}{c}\text { EORTC QLQ- } \\
\quad \text { C30 }\end{array}$ \\
\hline $\begin{array}{l}\text { Kim et al } 2011^{24} \\
N=70\end{array}$ & $25.5 \pm 21.2^{\ddagger}$ & $\begin{array}{l}\text { Strength } \\
\text { training } \\
\text { Sensory } \\
\text { training } \\
\text { Coordination } \\
\text { training }\end{array}$ & $\begin{array}{l}\text { Visual } \\
\text { Verbal }\end{array}$ & PFET & NR & I/week & 10 & $\begin{array}{c}\text { ARM } \\
\text { Wexner score } \\
\text { Daily stool frequency } \\
\text { satisfaction score }\end{array}$ & NR \\
\hline $\begin{array}{l}\text { Kuo et al } 2015^{27} \\
N=32\end{array}$ & NR & $\begin{array}{l}\text { Coordination } \\
\text { training }\end{array}$ & Visual & NMES & NR & 2-3/week & $8-12$ & $\begin{array}{c}\text { ARM } \\
\text { Wexner score }\end{array}$ & NR \\
\hline $\begin{array}{l}\text { Liang et al } 2016^{29} \\
\mathrm{~N}=61\end{array}$ & $7.7 \pm 2.9^{\ddagger}$ & $\begin{array}{l}\text { Strength } \\
\text { training } \\
\text { Sensory } \\
\text { training } \\
\text { Coordination } \\
\text { training }\end{array}$ & NR & NR & 30 & 2/day & NR & $\begin{array}{c}\text { ARM } \\
\text { Wexner score } \\
\text { Vaizey score }\end{array}$ & NR \\
\hline
\end{tabular}

Note: Values are median (range)*, mean $\pm \mathrm{SD}^{\ddagger}$.

Abbreviations: BF, Biofeedback; BFT, Biofeedback therapy; PEFT, Pelvic Floor Muscle Training; NR, Not reported; ARM, Anorectal Manometry; CCIS, Cleveland Clinic Incontinence Score; MSKCC, Memorial Sloan-Kettering Cancer Center bowel score; SF-36, Health Status and Fecal Incontinence Quality of Life score; FIQL, The Fecal Incontinence Quality of Life; NBM, The number of bowel movement; LARS, Low Anterior Resection Symptom; MCIS, Modified Cleveland Incontinence Score; EUS, Endoscopic Ultra Sonography; VAS, Visual analog scale; EORTC QLQ-C30, European Organization for Research and Treatment of Cancer Quality of Life Questionnaire Core 30; NMES,

Neuromuscular electrical stimulation. 


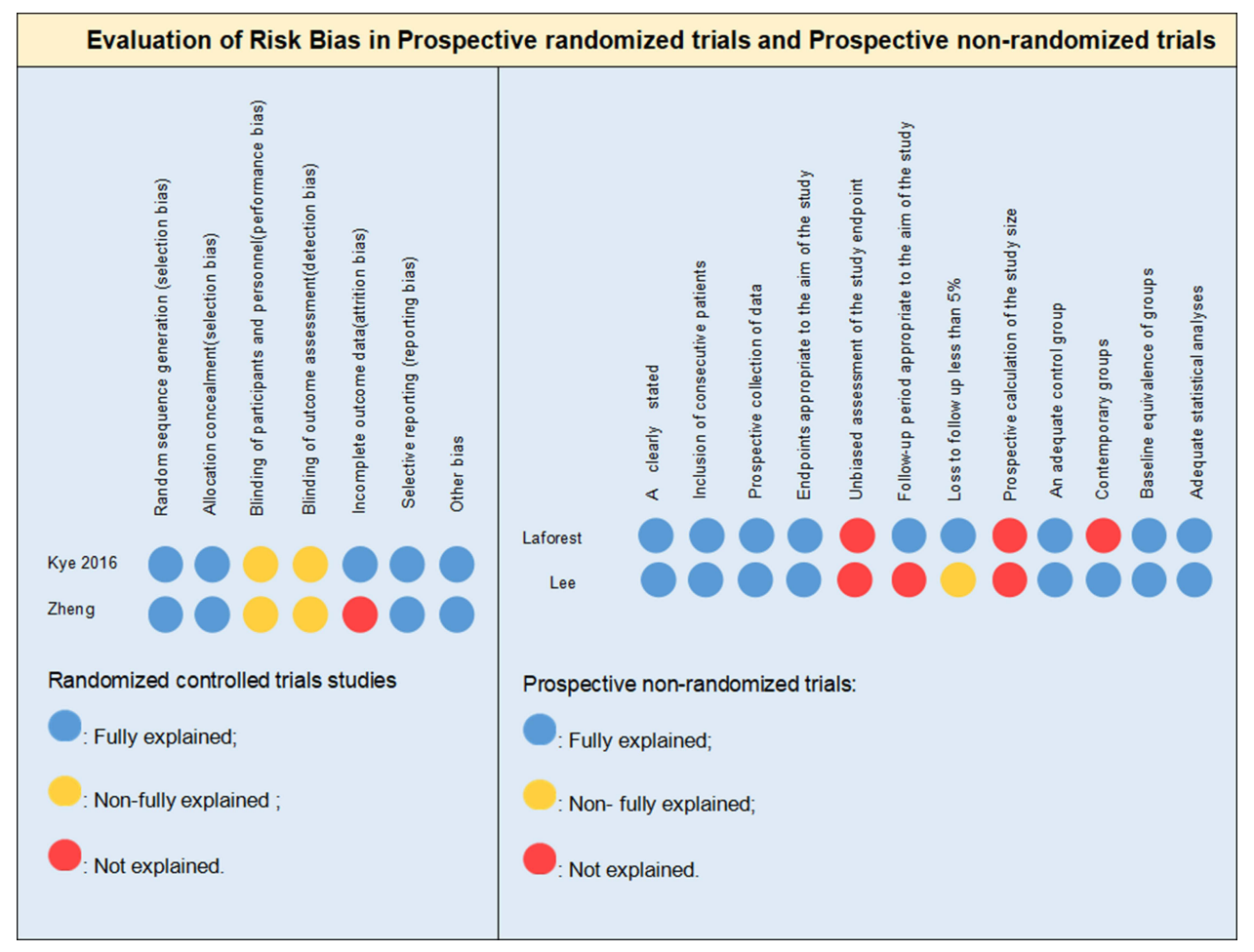

Figure 2 Evaluation of Risk Bias in Prospective randomized trials and Prospective non-randomized trials.

\section{Outcomes Characteristics}

The 12 included studies evaluated bowel function by subjective and objective measures and quality of life by questionnaires. Almost all studies evaluated subjective-feedback intestinal function through a gastrointestinal questionnaire (CCIS MSKCC LARS MCIS GS CGS), while 4 studies $^{22,24,26,28}$ evaluated that in terms of stool frequency, fecal incontinence episodes, and severity of fecal incontinence. Nine studies ${ }^{19-22,24,26-28,30}$ performed an ARM to evaluate anal function through objective indicators. In addition, one study performed an EUS examination to explore trends in the structure of the external anal sphincter (SE) and internal anal sphincter (SI). ${ }^{19}$ Three studies evaluated the quality of life using different questionnaires (EORTC QLQ-C30 FIQL SF-36). ${ }^{22,23,25}$ Table 2 displays the details.

\section{Risk of Bias Evaluation}

The risk of bias of studies is presented in Figures 2 and 3. Different questionnaire scales were adopted to evaluate different types of studies, using the Cochrane risk-of-bias (RoB) to evaluate the quality of RCT studies, using the MINORS scale to evaluate cohort studies, using the IHE scale to evaluate case series studies. Two independent reviewers (LHZ and GC) had no major differences during the evaluation process, and all disagreements were resolved. The majority of studies have made detailed statements about purpose and methods, patient selection process, intervention measures description, completeness of outcome indicators, and credibility. However, problems existed with the process of sample size calculation, whether blinding is operated or not, single-center studies, vague inclusion and exclusion criteria, insufficient follow-up, unclear source of support, and unexplained authors' contributions and relationships. In addition, an RCT study ${ }^{30}$ and a cohort study ${ }^{25}$ both show incomplete data. In terms of case series, 1 study $^{23}$ did not describe the interventions of interest and others.

\section{Outcomes}

\section{Objective Evaluation Outcome Measures}

ARM

Eight studies applied ARM to objectively evaluate intestinal function after rectal surgery. Allgayer et al ${ }^{19}$ believed that 


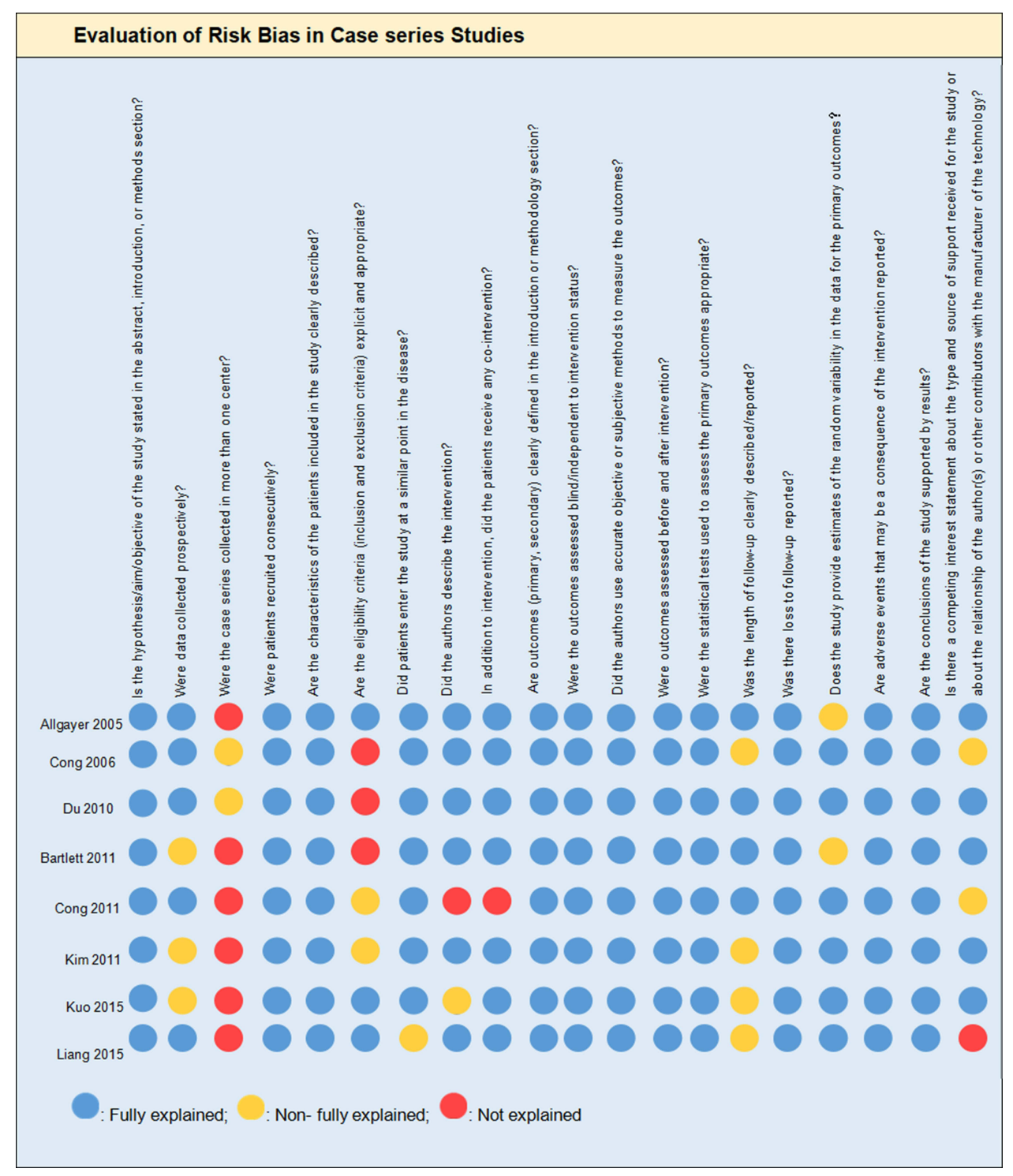

Figure 3 Evaluation of Risk Bias in Case series Studies.

ARM indicators were almost identical after biofeedback treatment, and there was no detailed presentation of the data and P-value. Most studies believed that after receiving biofeedback treatment, there is a significant improvement in the maximal resting pressure, maximum maximal squeeze pressure, and rectal capacity ( $\mathrm{P} \leq 0.05)$. However, there is no statistically significant difference in the sensory threshold. Two studies have analyzed mean resting pressure. As a result, $\mathrm{Du}$ et $\mathrm{al}^{21}$ believed that there was a statistical difference, while Kuo et $\mathrm{al}^{27}$ did not think so. When comparing the biofeedback treatment group and the control group (comparison between groups), only mean squeezing pressure and rectal capacity were considered to be statistically different.

\section{Subjective Evaluation Method}

Wexner Score

Two studies performed a comparison between the biofeedback treatment group and the control group. As a result, the biofeedback treatment group showed a lower Wexner score and better bowel function. The study of Laforest et $\mathrm{al}^{25}$ had statistical significance, while Lee et $\mathrm{al}^{26}$ did not. 


\section{Patient Diary}

Regarding the measurement of bowel function reported by patients, four studies showed that patients' reported incontinent episodes, bowel motions (per day), Bristol stool form scale, number of bowel movements/day, etc. improved after the intervention. Bartlett et $\mathrm{al}^{22}$ together with $\mathrm{Kim}^{24}$ showed statistical differences in Bowel motions (per day). Lee et $\mathrm{al}^{26}$ reported that there was no significant difference in defecation between the intervention group and the control group after biofeedback treatment. Kye et $\mathrm{al}^{28}$ did not perform baseline assessment, P-value, and also had no comparison with the control group.

\section{Patient Satisfaction}

Two articles followed up on patients' feedback satisfaction after biofeedback treatment. Unfortunately, none of the baseline assessment data was collected. Moreover, there was no control group for comparison, and no P-values were calculated. According to the analysis of existing data, the overall satisfaction score and the satisfaction with components of the biofeedback treatment were high.Table 3 displays the objective and subjective evaluation outcome measures.

\section{Quality of Life}

A total of 3 studies focused on the patients' quality of life. Three studies were respectively applied to FIQL, SF-36, and EORTC QLQ-C30 to evaluate the quality of life comprehensively from different perspectives. Table 4 displays the details.

FIQL

Bartlett et $\mathrm{a}^{22}$ followed up on 12 patients undergoing biofeedback treatment after rectal surgery for up to two years. After receiving biofeedback treatment for 2 years, the median values of lifestyle, coping, and embarrassment, etc., were further improved. Although the degree was not significant, it was statistically significant. Laforest et $\mathrm{al}^{25} \mathrm{compared} \mathrm{the}$ rehabilitation group with the control group at the same period. The results were as follows: regarding specific FIQL, the frequency of depression in the rehabilitation group was significantly reduced, and the self-perception was better, but there was no significant difference in the other 3 sub-indicators.

\section{SF-36}

Laforest et $\mathrm{al}^{25}$ believe that compared with the control group, biofeedback treatment combined with PFMT can significantly improve the overall quality of life, and statistical differences occurred in the vitality and mental function of SF-36.

\section{EORTC QLQ-C30}

Cong et $\mathrm{al}^{23}$ concluded that the changes in EORTC QLQ-C30 have no statistical significance in general. EORTC QLQC30 is a questionnaire developed to assess the quality of life of cancer patients in which higher scores in the fields of functional and general health status were associated with better functional status. Conversely, lower scores in the symptom domain were better. Compared with data before biofeedback treatment, patients score higher on diarrhea but lower on constipation and financial difficulties after treatment. There were no significant differences in the other 11 subindicators.

\section{Quantitative Synthesis}

Different studies have different outcome indicators to evaluate intestinal function. The Wexner score was used in 9 studies. $^{20-27,29}$ Two of them ${ }^{25,26}$ were excluded from quantitative synthesis because of insufficient data and the different types of study. The remaining 7 studies met the criteria for quantitative synthesis; the Wexner scores before and after biofeedback treatment can be meta-analyzed. Similarly, the Vaizey scores of the three studies ${ }^{20,21,29}$ can also be metaanalyzed.

In Figure 4, a meta-analysis of the Wexner score and the Vaizey score after Bio-feedback treatment is displayed. From the results of meta-analysis, it is easy to find that after biofeedback treatment, the Wexner score $\left(t^{*}=7, \mathrm{MD}=3.33 ; 95 \% \mathrm{CI}\right.$ 
Table 3 Results: Objective and Subjective Evaluation Outcome Measures

\begin{tabular}{|c|c|c|c|c|c|}
\hline Author and Year & $\mathbf{N}$ & Outcomes & $\begin{array}{l}\text { Pre-Biofeedback Therapy } \\
\text { Mean } \pm \text { SD/Median (range) }\end{array}$ & $\begin{array}{l}\text { Post-Biofeedback Therapy } \\
\text { Mean } \pm \text { SD/ Median (Range) }\end{array}$ & P-value \\
\hline $\begin{array}{l}\text { Objective evaluation methoc } \\
\text { ARM } \\
\text { Allgayer et al } 2005^{19} \\
\text { Irradiated } \\
\text { Non-irradiated }\end{array}$ & 41 & $\begin{array}{l}\text { Resting pressure }(\mathrm{mmHg}) \\
\text { Squeeze pressure }(\mathrm{mmHg}) \\
\text { Sphincter length }(\mathrm{cm}) \\
\text { Sensation threshold }(\mathrm{mL}) \\
\text { Pain threshold }(\mathrm{mL}) \\
\text { Resting pressure }(\mathrm{mmHg}) \\
\text { Squeeze pressure }(\mathrm{mmHg}) \\
\text { Sphincter length }(\mathrm{cm}) \\
\text { Sensation threshold }(\mathrm{mL}) \\
\text { Pain threshold }(\mathrm{mL})\end{array}$ & $\begin{array}{c}27.3 \pm 17.2 \\
79.5 \pm 34.0 \\
2.5 \pm 0.5 \\
44.7 \pm 16.9 \\
91.2 \pm 36.8 \\
33.3 \pm 17.8 \\
79.5 \pm 34.1 \\
2.4 \pm 0.6 \\
43.0 \pm 13.8 \\
103.0 \pm 29.5\end{array}$ & Almost identical & NR \\
\hline Cong et al $2006^{20}$ & 16 & $\begin{array}{l}\text { The maximal resting pressure }(\mathrm{mmHg}) \\
\text { The maximal contraction pressure }(\mathrm{mmHg}) \\
\text { Contraction vector volume }(\mathrm{cm} \times \mathrm{mmHg}) \\
\text { Resting vector volume }(\mathrm{cm} \times \mathrm{mmHg})\end{array}$ & $\begin{array}{c}65.6 \pm 13.3 \\
\mid 43.6 \pm 46.5 \\
|33337.0 \pm 749| . \mid \\
509.2 \pm 95.0\end{array}$ & $\begin{array}{c}66.6 \pm 13.0 \\
205.6 \pm 44.5 \\
50664.6 \pm 8040.1 \\
516.5 \pm 96.0\end{array}$ & $\begin{array}{l}0.002 \\
<0.001 \\
<0.001 \\
0.001\end{array}$ \\
\hline Du et al $2010^{2 l}$ & 24 & $\begin{array}{l}\text { Resting pressure }(\mathrm{mmHg}) \\
\text { Maximum squeeze pressure }(\mathrm{mmHg}) \\
\text { Sensory threshold }(\mathrm{mL}) \\
\text { Maximum tolerated volume }(\mathrm{mL})\end{array}$ & $\begin{array}{c}27.8 \pm 9.0 \\
118.3 \pm 42.9 \\
19.0 \pm 6.1 \\
97.5 \pm 52.8\end{array}$ & $\begin{array}{c}47.9 \pm 9.3 \\
193.2 \pm 38.2 \\
21.5 \pm 4.8 \\
189.1 \pm 39.0\end{array}$ & $\begin{array}{l}<0.01 \\
<0.01 \\
0.101 \\
<0.01\end{array}$ \\
\hline
\end{tabular}




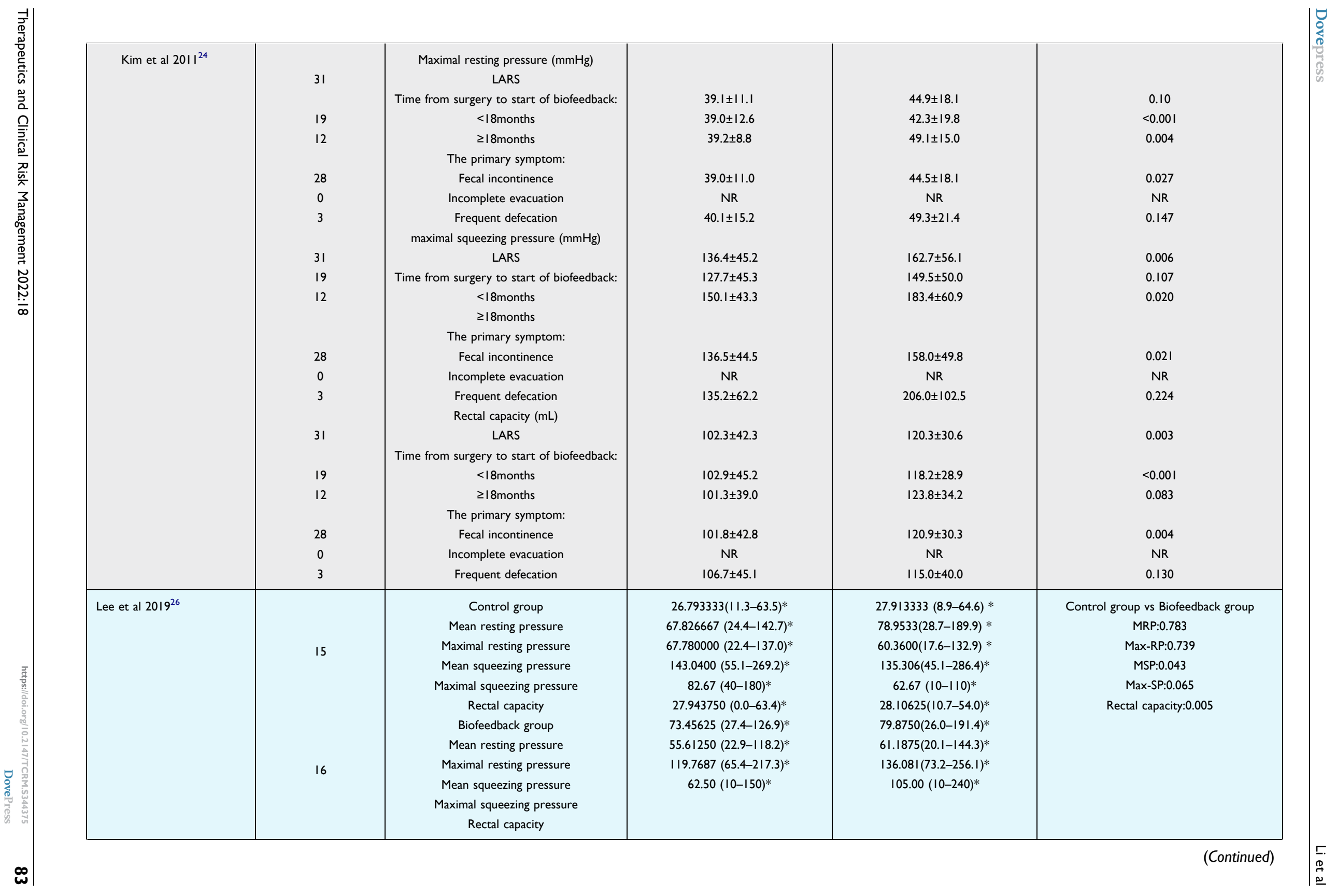


Table 3 (Continued).

\begin{tabular}{|c|c|c|c|c|c|}
\hline Author and Year & $\mathbf{N}$ & Outcomes & $\begin{array}{l}\text { Pre-Biofeedback Therapy } \\
\text { Mean } \pm \text { SD/Median (range) }\end{array}$ & $\begin{array}{l}\text { Post-Biofeedback Therapy } \\
\text { Mean } \pm \text { SD/ Median (Range) }\end{array}$ & P-value \\
\hline Kuo et al $2015^{27}$ & 32 & $\begin{array}{l}\text { Resting pressure }(\mathrm{hPa}) \\
\text { Maximal squeeze pressure }(\mathrm{hPa}) \\
\text { Resting electromyography }(\mathrm{hPa}) \\
\text { Maximal squeeze EMG }(\mathrm{hPa})\end{array}$ & $\begin{array}{c}-4.21 \pm 7.29 \\
34.32 \pm 35.37 \\
-0.47 \pm 2.21 \\
28.37 \pm 16.75\end{array}$ & $\begin{array}{c}-4.08 \pm 3.80 \\
37.08 \pm 22.42 \\
-0.58 \pm 5.67 \\
30.79 \pm 16.00\end{array}$ & $\begin{array}{l}0.061 \\
0.014 \\
0.760 \\
0.990\end{array}$ \\
\hline Kye et al $2016^{28}$ & 26 & $\begin{array}{c}\text { Control group } \\
\text { Mean resting pressure } \\
\text { Maximal squeezing pressure } \\
\text { Rectal compliance } \\
\text { Rectal sensory threshold } \\
\text { Biofeedback group } \\
\text { Mean resting pressure } \\
\text { Maximal squeezing pressure } \\
\text { Rectal compliance } \\
\text { Rectal sensory threshold }\end{array}$ & $N R$ & $\begin{array}{l}0.79 \pm 0.4 \\
0.83 \pm 0.4 \\
0.82 \pm 0.7 \\
0.77 \pm 0.5 \\
\\
0.88 \pm 0.7 \\
0.74 \pm 0.3 \\
0.93 \pm 0.7 \\
0.66 \pm 0.4\end{array}$ & $\begin{array}{c}\text { Control group } \\
\text { vs } \\
\text { Biofeedback } \\
\text { group } \\
\text { MRP:0.6I2 } \\
\text { Max-SP: } \\
0.445 \\
\text { Rectal } \\
\text { compliance: } 0.928 \\
\text { RST:0.438 }\end{array}$ \\
\hline Liang et al $2016^{29}$ & 61 & $\begin{array}{l}\text { Maximal resting pressure } \\
\text { Maximal squeezing pressure } \\
\text { Rectal Sensitivity } \\
\text { Rectal Capacity }\end{array}$ & $\begin{array}{l}25.8 \pm 12.3 \\
120.2 \pm 42.0 \\
23.4 \pm 12.7 \\
119.0 \pm 50.7\end{array}$ & $\begin{array}{l}37.0 \pm 12.8 \\
146.5 \pm 40.9 \\
27.4 \pm 12.5 \\
143.6 \pm 52.8\end{array}$ & $\begin{array}{l}<0.001 \\
0.001 \\
0.089 \\
0.015\end{array}$ \\
\hline $\begin{array}{l}\text { Subjective evaluation method } \\
\text { Wexner } \\
\text { Laforet al } 2012^{25} \\
\text { Lee et al } 2019^{26}\end{array}$ & $\begin{array}{l}22 \\
24 \\
15 \\
16\end{array}$ & $\begin{array}{l}\text { Rehabilitation group } \\
\text { Control group } \\
\text { Control group } \\
\text { Biofeedback group }\end{array}$ & $\begin{array}{c}\text { NR } \\
\text { NR } \\
10.47(7-13) * \\
13.06(6-16) *\end{array}$ & $\begin{array}{c}8.3 \pm 3.9 \\
9.9 \pm 3.0 \\
8.53(4-12)^{*} \\
6.81(3-10)^{*}\end{array}$ & Control group vs Rehabilitation group:0.100 \\
\hline
\end{tabular}




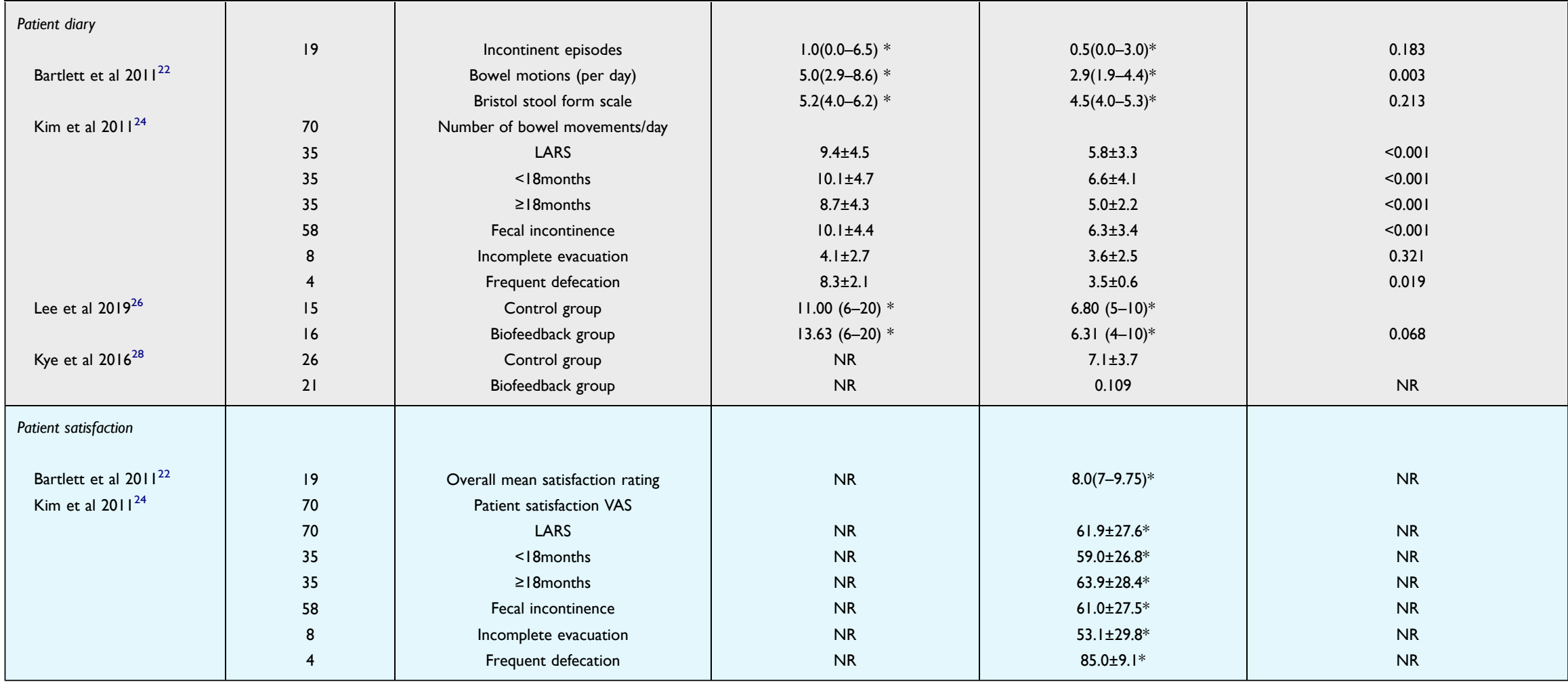

Note: Values in parentheses are mean \pm SD unless indicated otherwise; values is median (range)*.

Abbreviations: LARS, Low Anterior Resection Symptom; NR, not reported. 
Table 4 Results: Quality of Life Outcome Measures

\begin{tabular}{|c|c|c|c|c|c|}
\hline $\begin{array}{l}\text { Quality of life } \\
\text { FIQL } \\
\text { Bartlett et al } 2011^{22} \\
\text { Laforest et al } 2012^{25}\end{array}$ & . & $\begin{array}{c}\text { Lifestyle } \\
\text { Coping } \\
\text { Depression } \\
\text { Embarrassment } \\
\text { Rehabilitation group } \\
\text { Lifestyle } \\
\text { Coping/Behaviour } \\
\text { Depression/Self-perception } \\
\text { Embarrassment } \\
\text { Control group } \\
\text { Lifestyle } \\
\text { Coping/Behaviour } \\
\text { Depression/Self-perception } \\
\text { Embarrassment }\end{array}$ & $\begin{array}{c}2.8(2.1-3.7)^{*} \\
2.1(1.7-3.0) * \\
3.4(2.6-3.7) * \\
3.0(1.7-3.0) * \\
\\
\text { NR } \\
\text { NR } \\
\text { NR } \\
\text { NR } \\
\text { NR } \\
\text { NR } \\
\text { NR } \\
\text { NR }\end{array}$ & $\begin{array}{l}3.5(3.0-4.0) * \\
2.9(2.3-3.5) * \\
3.3(3.0-3.6) * \\
3.3(2.7-4.0) * \\
\\
2.6 \pm 0.9 \\
2.2 \pm 0.8 \\
3.2 \pm 0.6 \\
2.5 \pm 0.7 \\
2.3 \pm 0.9 \\
2.0 \pm 0.8 \\
2.6 \pm 0.7 \\
2.4 \pm 0.9\end{array}$ & $\begin{array}{c}0.001 \\
0.001 \\
0.828 \\
0.001 \\
\\
\\
\text { Between Group } \\
\text { Lifestyle:0.27 } \\
\text { Coping/Behavi } \\
\text { our:0.56 } \\
\text { Depression/Self-perception:0.0 } \\
05 \\
\text { Embarrassmen } \\
\text { t:0.64 }\end{array}$ \\
\hline $\begin{array}{l}\text { SF-36 } \\
\quad \text { Laforest et al } 2012^{25}\end{array}$ & 24 & $\begin{array}{c}\text { Rehabilitation group } \\
\text { Physical functioning } \\
\text { Role limitations - physical } \\
\text { Bodily pain } \\
\text { General health } \\
\text { Vitality } \\
\text { Social functioning } \\
\text { Role limitations - emotional } \\
\text { Mental health } \\
\text { Physical functioning } \\
\text { Mental functioning } \\
\text { Control group } \\
\text { Physical functioning } \\
\text { Role limitations -physical } \\
\text { Bodily pain } \\
\text { General health } \\
\text { Vitality } \\
\text { Social functioning } \\
\text { Role limitations - emotional } \\
\text { Mental health } \\
\text { Physical functioning } \\
\text { Mental functioning }\end{array}$ & $\begin{array}{l}\text { NR } \\
N R \\
N R \\
N R \\
N R \\
N R \\
N R \\
N R \\
N R \\
N R \\
\\
N R \\
N R \\
N R \\
N R \\
N R \\
N R \\
N R \\
N R \\
N R \\
N R\end{array}$ & $\begin{array}{c}49.1 \pm 6.8 \\
40.0 \pm 12.4 \\
48.1 \pm 11.2 \\
46.5 \pm 9.0 \\
47.3 \pm 9.9 \\
42.0 \pm 10.4 \\
43.5 \pm 11.0 \\
44.2 \pm 12.4 \\
48.8 \pm 8.5 \\
48.3 \pm 7.1 \\
47.4 \pm 7.4 \\
39.3 \pm 9.7 \\
48.7 \pm 9.6 \\
43.0 \pm 7.8 \\
39.3 \pm 8.2 \\
45.6 \pm 10.2 \\
43.0 \pm 12.5 \\
44.0 \pm 9.5 \\
46.0 \pm 6.7 \\
42.7 \pm 8.6\end{array}$ & $\begin{array}{c}\text { Between Group } \\
\text { Physical functioning:0.4 } \\
6 \\
\text { Role limitations } \\
\text {-physical:0.97 } \\
\text { Bodily } \\
\text { pain: } 0.8 \text { I } \\
\text { General } \\
\text { health:0.17 } \\
\text { Vitality:0.004 } \\
\text { Social } \\
\text { functioning:0.2 } \\
4 \\
\text { Role limitations } \\
- \\
\text { emotional:0.92 } \\
\text { Mental } \\
\text { health:0.84 } \\
\text { Physical } \\
\text { functioning:0.2 } \\
\text { I } \\
\text { Mental functioning:0.0 } \\
2\end{array}$ \\
\hline
\end{tabular}




\begin{tabular}{|c|c|c|c|c|c|}
\hline $\begin{array}{l}\text { EORTC QLQ-C30 } \\
\text { Cong et al } 2011^{23}\end{array}$ & 11 & $\begin{array}{l}\text { Functional scales } \\
\text { Physical functioning } \\
\text { Role functioning } \\
\text { Cognitive functioning } \\
\text { Emotional functioning } \\
\text { Social functioning } \\
\text { Symptom scales / items } \\
\text { Fatigue } \\
\text { Nausea and vomiting } \\
\text { Pain } \\
\text { Dyspepsia } \\
\text { Sleep disturbance } \\
\text { Appetite loss } \\
\text { Constipation } \\
\text { Diarrhoea } \\
\text { Financial difficulties }\end{array}$ & $\begin{array}{l}80.3 \pm 13.6 \\
61.4 \pm 21.8 \\
70.3 \pm 18.6 \\
56.8 \pm 12.5 \\
50.4 \pm 11.6 \\
38.6 \pm 5.4 \\
37.6 \pm 4.3 \\
18.7 \pm 2.1 \\
20.3 \pm 4.1 \\
31.5 \pm 2.2 \\
30.8 \pm 2.7 \\
13.1 \pm 2.7 \\
64.4 \pm 5.2 \\
21.7 \pm 3.8\end{array}$ & $\begin{array}{l}75.3 \pm 12.1 \\
55.8 \pm 20.5 \\
65.9 \pm 15.8 \\
52.5 \pm 14.2 \\
44.4 \pm 10.3 \\
40.6 \pm 4.7 \\
43.8 \pm 3.6 \\
21.1 \pm 2.2 \\
22.9 \pm 1.3 \\
34.8 \pm 3.6 \\
32.6 \pm 3.1 \\
17.7 \pm 2.4 \\
53.7 \pm 6.0 \\
28.8 \pm 2.0\end{array}$ & $\begin{array}{l}0.674 \\
0.752 \\
0.782 \\
0.689 \\
0.580 \\
\\
\\
0.595 \\
0.067 \\
0.163 \\
0.273 \\
0.199 \\
0.410 \\
0.041 \\
0.039 \\
0.015\end{array}$ \\
\hline
\end{tabular}

Note: Values in parentheses are mean \pm SD unless indicated otherwise; values is median (range)*.

Abbreviations: SF-36, Health Status and Fecal Incontinence Quality of Life score; FIQL, The Fecal Incontinence Quality of Life; EORTC QLQ-C30, European Organization for Research and Treatment of Cancer Quality of Life Questionnaire Core 30; NR, not reported. 


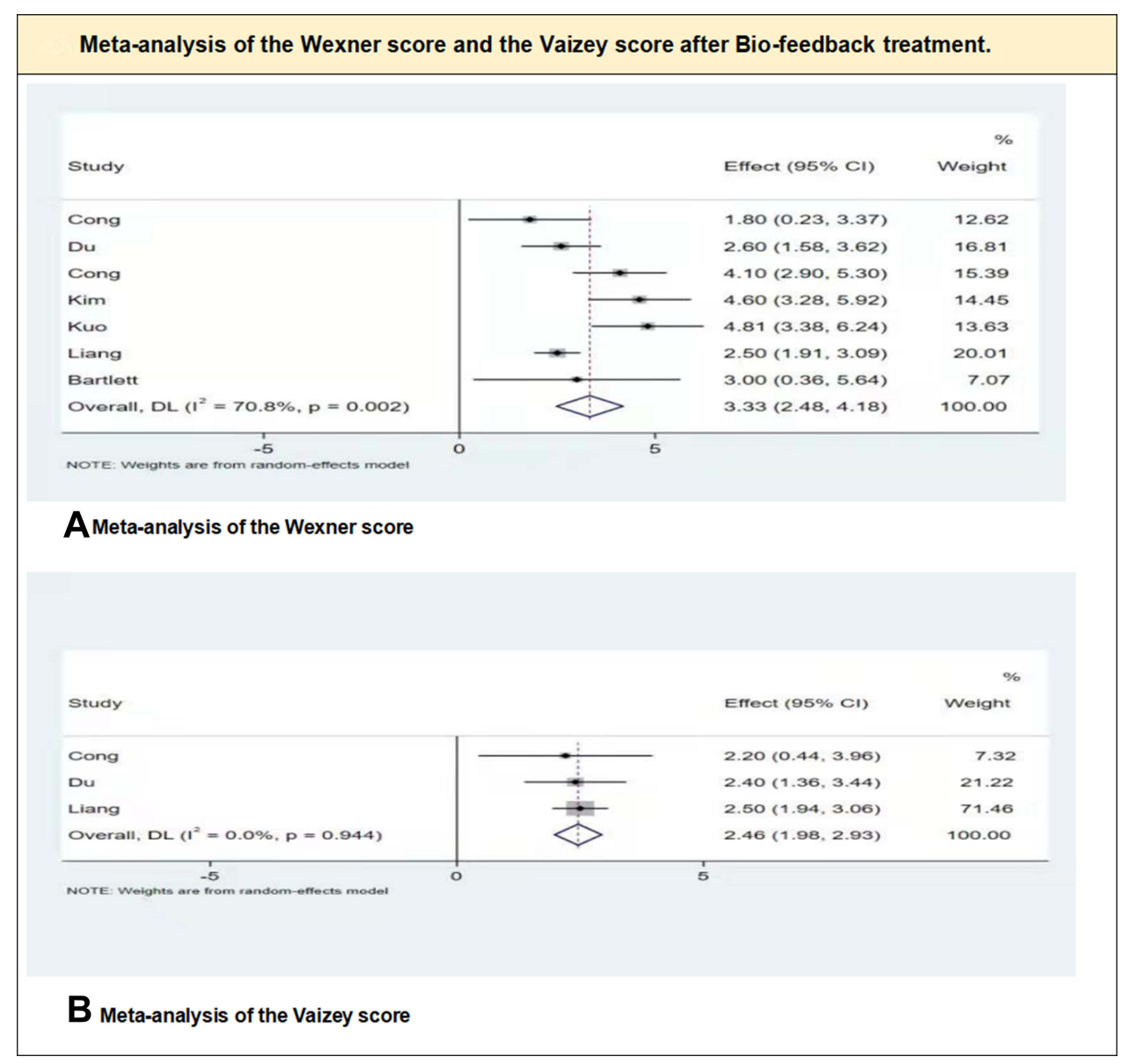

Figure 4 Meta-analysis of the Wexner score(A) and the Vaizey score(B) after Bio-feedback treatment.

Notes: Forest plot of all studies included for this meta-analysis with pooled standardized mean difference for Wexner and Vaizey score.Values represent effect sizes (weighted mean differences) and $95 \%$ confidence intervals. The pooled effect sizes were calculated using a random effects model.

$[2.48,4.18]$, Figure 4A) and Vaizey score $\left(\mathrm{t}^{*}=3, \mathrm{MD}=2.46 ; 95 \% \mathrm{CI}[1.98,2.93]\right.$, Figure 4B) of patients with bowel dysfunction after rectal cancer surgery were significantly decreased. It indicates that bowel function was significantly improved. There is significant heterogeneity in the Wexner score $\left(\mathrm{I}_{2}=70.8 \%, \mathrm{p}=0.002, \mathrm{H}=1.850\right)$, while no evidence shows that the Vaizey score is heterogeneous $\left(I_{2}=0.0 \%, p=0.944, H=0.240\right)$. Therefore, we performed subgroup analysis stratified by whether to conduct electrical stimulation, adjuvant chemoradiotherapy, and surgery approach. Note:" $t$ "is the number of included studies.*

Two of the seven studies were included in the Wexner score meta-analysis that used biofeedback therapy together with electrical stimulation therapy ${ }^{20,21}(\mathrm{t}=3, \mathrm{MD}=2.36 ; 95 \% \mathrm{CI}[1.51,3.22]$, Figure 5A) while patients of five studies did not receive electrical stimulation ${ }^{22-24,27,29}(\mathrm{t}=4, \mathrm{MD}=3.79$; 95\% CI [2.66, 4.93], Figure 5A); Patients in three studies $^{20-22}$ did not receive adjuvant chemoradiotherapy $\left(\mathrm{t}=3, \mathrm{MD}=2.42 ; 95 \% \mathrm{CI}[1.61,3.24]\right.$, Figure 5B); one study ${ }^{23}$ performed chemotherapy and radiotherapy for all patients $(\mathrm{t}=1, \mathrm{MD}=4.10$; 95\% CI [2.90, 5.30], Figure 5B); two studies $^{24,29}$ performed radiotherapy on parts of patients, and others ${ }^{27}$ received chemotherapy $(\mathrm{t}=2, \mathrm{MD}=3.46 ; 95 \% \mathrm{CI}$ [1.41, 5.51], Figure 5B); one study only performed chemotherapy on patients $(\mathrm{t}=1, \mathrm{MD}=4.81 ; 95 \% \mathrm{CI}[3.38,6.24]$, Figure 5B); Different studies performed different approaches of surgery for patients, Two studies ${ }^{20,27}$ performed ISR $(\mathrm{t}=$ $2, \mathrm{MD}=3.32 ; 95 \% \mathrm{CI}[0.37,6.27]$, Figure $5 \mathrm{C})$; four studies ${ }^{21,22,24,29}$ performed $\mathrm{AR}(\mathrm{t}=4, \mathrm{MD}=3.08 ; 95 \% \mathrm{CI}[2.12$, 4.04], Figure 5C); one study performed transabdominal and transanal Surgical PLRAS surgery ${ }^{23}(t=1, M D=4.10 ; 95 \%$ CI [2.90, 5.30], Figure 5C).Changes in heterogeneity between groups are reflected by $\mathrm{I}_{2}$. 


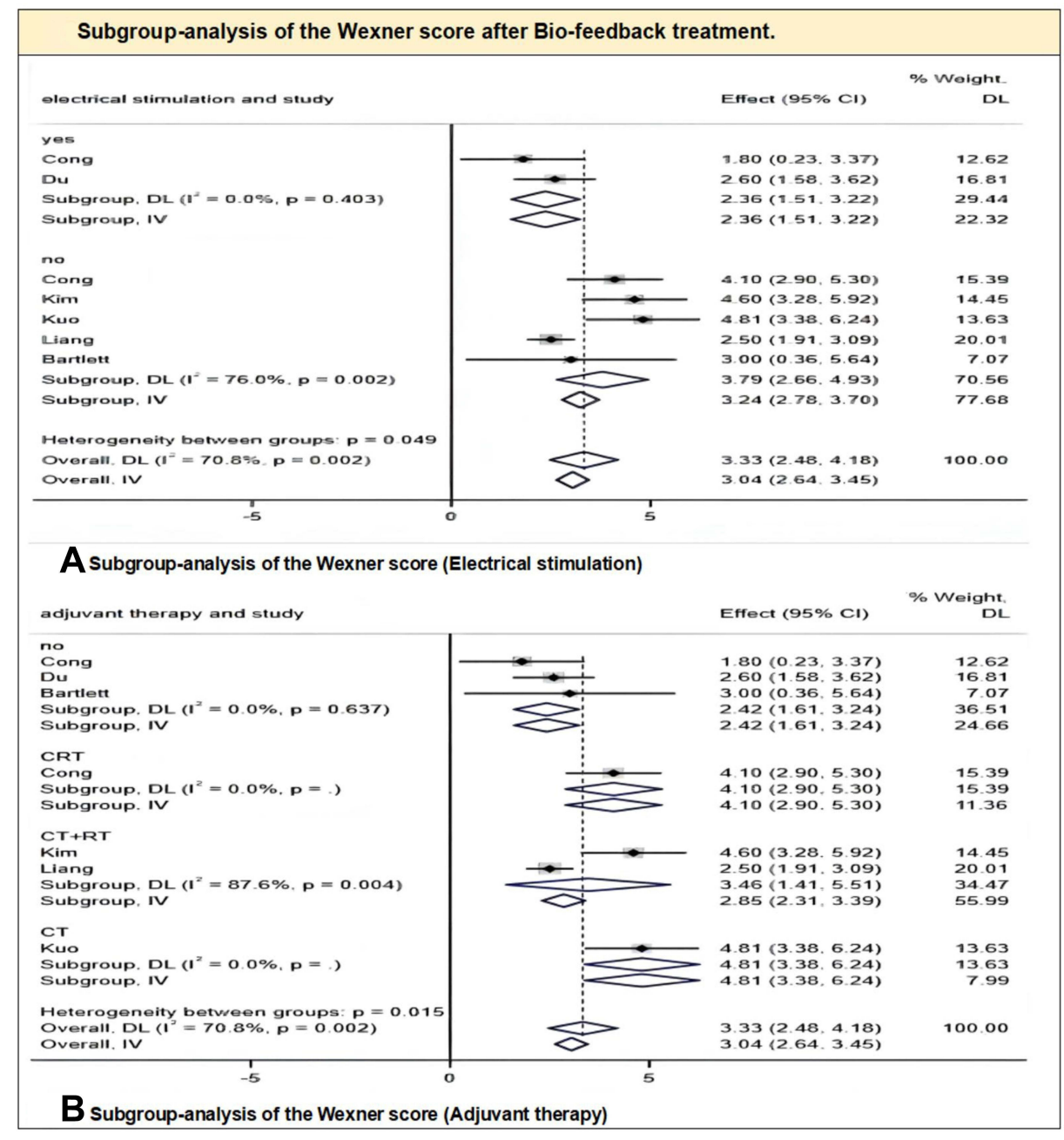

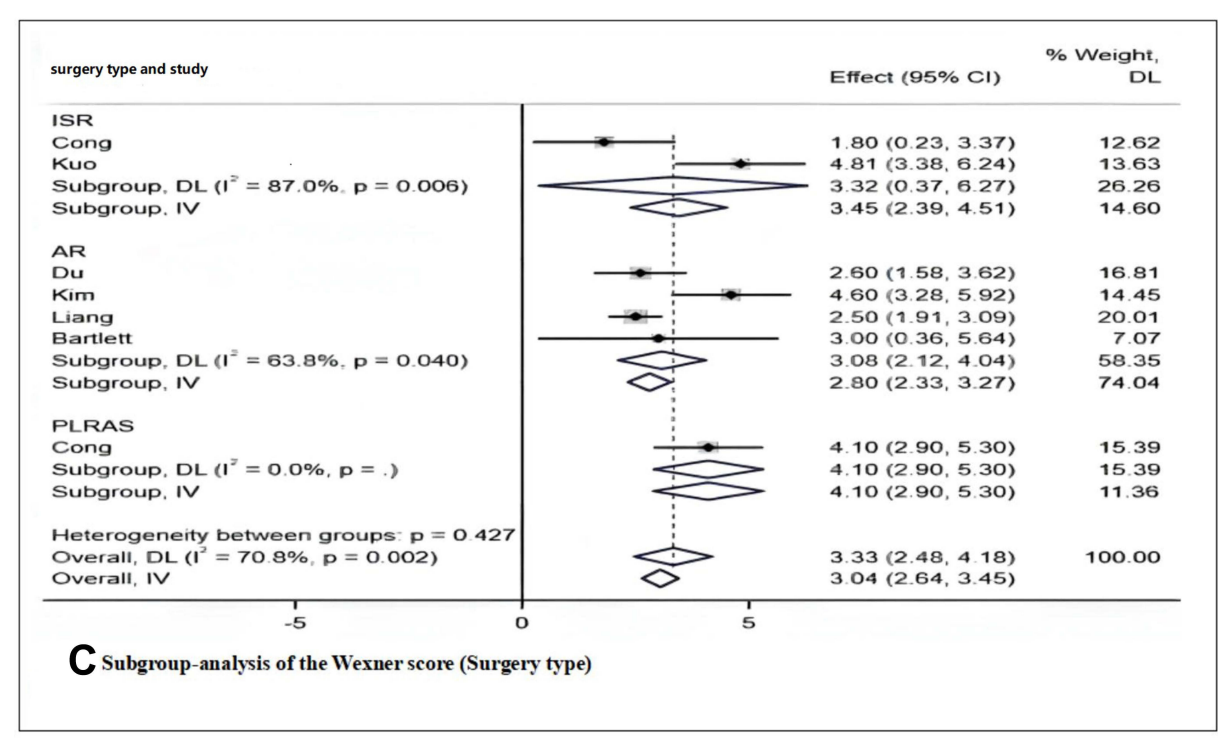

Figure 5 Sub-group analysis of the Wexner score for Electrical stimulation (A), Adjuvant therapy(B), and Surgery type(C)after Bio-feedback treatment.

Notes: Forest plot for Wexner and Vaizey scores in the subgroup analysis.Values represent effect sizes (weighted mean differences) and $95 \%$ confidence intervals. 


\section{Discussion}

This review aims to analyze and explore the evidence for the effect of biofeedback therapy on patients with bowel dysfunction after surgery. Based on the existing studies, the patient's bowel function has recovered after biofeedback treatment, and the overall quality of life has improved. ${ }^{31}$ Limited to fewer RCTs in related fields, the comparison of intestinal function and quality of life between the intervention group and the control group still needs further research. It reflects our urgent need for more good-quality RCTs in the field, which may provide higher-level evidence to clarify whether biofeedback treatment should be performed to improve bowel function and promote quality of life.

Some published systematic reviews hold the opinion that biofeedback therapy alone as well as PFMT mixed with biofeedback therapy show a positive effect in improving intestinal function. The conclusion is the same as in our review, which affirms the positive effect of biofeedback therapy. ${ }^{32,33}$

The conclusion requires careful analysis. Recent studies have indicated that the distance from the lower end to the dentate line, the height of the anastomosis, and whether a defunctioning stoma is performed are risk factors for postoperative bowel dysfunction in patients with rectal cancer. However, the data in these aspects is missing various degrees, so it is difficult for us to perform subgroup analysis or quantitative synthesis of these heterogeneous indicators. ${ }^{34}$ The factor of electrical stimulation and adjuvant therapy accounted for the inter-study heterogeneity while surgery type did not, which was consistent with the result of subgroup analysis. When mentioning surgery type, it is worth recalling that patients underwent a new surgical procedure (PLRAS), ${ }^{23}$ which is indicated for rectal adenocarcinomas located below the dentate line or with external sphincter muscle invasion. Tumor height is closely related to the surgical approach. Most patients who underwent ISR surgery had a distance of $<5 \mathrm{~cm}$ from the lower end to the dentate line, whereas patients who underwent other procedures were $\geq 5 \mathrm{~cm}$. What is more, we have no way to assess the baseline level of bowel function before surgery. Some of the patients suffered more severe bowel dysfunction before surgery, which led to the evaporation of a certain degree of credibility in bowel function changes.

Some studies used ARM and EUS to evaluate the anorectal function objectively. Seven studies believe that varying degrees of improvement occurred in different sub-indicators after biofeedback treatment, which is trustworthy. Although different centers implement different ARM equipment and set different values for parameters, resulting in data that cannot accord well with each other, the risk of bias is reduced after converting to the changes before and after treatment. Although there is no statistical difference because of the small sample size, Allgayer et al ${ }^{19}$ used EUS as an evaluation index of postoperative bowel function. The effectiveness of biofeedback therapy combined with PFMT in the postoperative bowel function of this review is consistent with the results of the systematic review conducted by Maris et al. ${ }^{33}$ The present results indicate that patients' reported incident episodes, bowel motions (per day), and number of bowel movements/day improved properly after biofeedback treatment. Overall, it is hard to tell whether the positive effect of biofeedback treatment on the patients' reported bowel function measurement is the result of natural recovery or the Hawthorne effect. ${ }^{35}$ In our review, few studies performed dynamic and continuous follow-up evaluations on patients' bowel function after biofeedback treatment which means their variation trend cannot be assessed. Therefore, we urgently need a randomized controlled trial in longitudinal follow-up to explore whether the long-term efficacy of biofeedback therapy can be maintained or not. Due to the lack of data, we cannot draw any valid conclusions on patients' satisfaction with bowel function.

Only three studies focused on changes in quality of life, and different questionnaires were used to evaluate different aspects of the quality of life. The sample size is small. The study by Bartlett et $\mathrm{al}^{22}$ has a long follow-up interval of up to 2 years. There are too many interfering factors during this period, so positive results are not credible. Therefore, we should pay more attention to the quality of life of those with intestinal dysfunction, and we also need large sample sizes and high-quality RCTs with high-frequency follow-up.

After integrating studies that can be quantitative synthesis, meta analysis of Wexner score shows highly heterogeneous results, which may be related to patients who underwent biofeedback therapy combined with PFMT and electrical stimulation therapy. PFMT is recommended as an early intervention in the 5th International Consultation on Incontinence, which is considered to positively affect the treatment of fecal incontinence. ${ }^{36}$ So it is hard to say whether the Wexner score's recovery is the result of biofeedback treatment, PFMT, and electrical stimulation together or separately. Almost all studies that can be meta-analyzed were performed using PFMT, so we can only perform subgroup 
analysis on electrical stimulation treatment. The duration and frequency of biofeedback treatment affect the dosage and efficacy of biofeedback therapy. ${ }^{37}$ It is necessary to carry out more high-quality randomized controlled trials to determine the primary treatment option for biofeedback therapy and provide guidance for clinicians caring for this population in clinical practice.

\section{Study Limitation}

The main limitations of this review lie in the following aspects: 1. Selection bias: Although our studies have no language restrictions, we did not conduct literature searches in specialized databases in other countries. This may result in possible selection bias. 2. Lacking randomized controlled trial studies. At the same time, few included studies finished explaining whether to blind, and the data opacity also existed. Most studies are case series or cohort studies, with no high-quality control group, and no longitudinal studies. 3. We hope for more quantitative synthesis and meta-analysis to draw a watertight conclusion. However, in addition to the Wexner score and Vaizey score, it is difficult to find a unified outcome index to evaluate intestinal function. 4. Most patients were treated with PFMT while undergoing biofeedback treatment, so there is no way to perform subgroup analysis on patients treated with PFMT. Therefore, this systematic review may not tell us the therapeutic effect of biofeedback treatment, but it will show the effect of biofeedback therapy combined with various treatments. 5 . None of the studies reported adverse events related to training.

\section{Conclusion}

Although biofeedback therapy may improve intestinal function, quality of life as well as anal function reflected by ARM after surgery, patients' satisfaction is still unclear. However, the data was obtained from small studies with a high risk of bias and the conclusion must be interpreted with caution. This intervention can be viewed as being in the development phase of research. ${ }^{38,39}$ At the same time, more good-quality research is necessary to explore the best biofeedback treatment options (single duration/quality frequency/length of treatment time), so that biofeedback therapy can be applied to clinical practice more accurately.

\section{Abbreviations}

RCTs, randomized controlled trials studies; ARM, Anorectal Manometry; ISR, Intersphincteric Resection; AR, Anterior Resection; PLRAS, Partial Longitudinal Resection of the Anorectum and Sphincter; LAR, Low Anterior Resection; ULAR, Ultra Low Anterior Resection; TME, Total Mesorectal Excision; TaTME, Transanal Total Mesorectal Excision; PFMT, Pelvic Floor Muscle Training; EUS, Endoscopic Ultra Sonography.

\section{Funding}

This study was supported by National Science and Technology Support Programme Project (2015BAI13B09), National Science and Technology Support Programme Project (2015BAI13B09).

\section{Disclosure}

The authors declared no conflicts of interest.

\section{References}

1. Sung H, Ferlay J, Siegel RL, et al. Global cancer statistics 2020: GLOBOCAN estimates of incidence and mortality worldwide for 36 cancers in 185 countries. CA Cancer J Clin. 2021;71:209-249. doi:10.3322/caac.21660

2. Siegel RL, Miller KD, Goding Sauer A, et al. Colorectal cancer statistics, 2020. CA Cancer J Clin. 2020;70:145-164. doi:10.3322/caac.21601

3. Chawla N, Butler EN, Lund J, et al. Patterns of colorectal cancer care in Europe, Australia, and New Zealand. J Natl Cancer Inst Monogr. 2013;2013:36-61. doi:10.1093/jncimonographs/lgt009

4. Damin DC, Lazzaron AR. Evolving treatment strategies for colorectal cancer: a critical review of current therapeutic options. World J Gastroenterol. 2014;20:877-887. doi:10.3748/wjg.v20.i4.877

5. Bryant CL, Lunniss PJ, Knowles CH, et al. Anterior resection syndrome. Lancet Oncol. 2012;13:e403-e408. doi:10.1016/S1470-2045(12)70236-X

6. Sloots K, Bartlett L. Practical strategies for treating postsurgical bowel dysfunction. J Wound Ostomy Contin Nurs. 2009;36:522-527. doi:10.1097/ WON.0b013e3181b35e95 
7. Sloots K, Bartlett L, Ho YH. Treatment of postsurgery bowel dysfunction: biofeedback therapy. J Wound Ostomy Contin Nurs. 2009;36:651-658. doi:10.1097/WON.0b013e3181bd8811

8. Tate JJ, Milner CE. Real-time kinematic, temporospatial, and kinetic biofeedback during gait retraining in patients: a systematic review. Phys Ther. 2010;90:1123-1134. doi:10.2522/ptj.20080281

9. Giggins OM, Persson UM, Caulfield B. Biofeedback in rehabilitation. J Neuroeng Rehabil. 2013;10:60. doi:10.1186/1743-0003-10-60

10. Zhang Z, Wu H, Wang W, Wang B. A smartphone based respiratory biofeedback system. In Proceedings of The Third International Conference on Biomedical Engineeringand Informatics. Yantai, China: IEEE; 2010.

11. Panic N, Leoncini E, de Belvis G, Ricciardi W, Boccia S. Evaluation of the endorsement of the preferred reporting items for systematic reviews and meta-analysis (PRISMA)statement on the quality of published systematic review and meta-analyses. PLoS One. 2013;8:e83138. doi:10.1371/ journal.pone. 0083138

12. Moher D, Liberati A, Tetzlaff J, Altman DG; PRISMA Group. Preferred reporting items for systematic reviews and meta-analyses: the PRISMA statement. PLoS Med. 2009;6:e1000097. doi:10.1371/journal.pmed.1000097

13. Buchter RB, Weise A, Pieper D. Development, testing and use of data extraction forms in systematic reviews: a review of methodological guidance. BMC Med Res Methodol. 2020;20:259. doi:10.1186/s12874-020-01143-3

14. Sterne JAC, Savovic J, Page MJ, et al. RoB 2: a revised tool for assessing risk of bias in randomised trials. BMJ. 2019;366:14898. doi:10.1136/bmj. 14898

15. Slim K, Nini E, Forestier D, Kwiatkowshi F, Panis Y, Chipponi J. Methodological index for non-randomized studies (MINORS): development and validation of a new instrument. ZNZ J Surg. 2003;73:712-716.

16. Mago C, Guo B, Schopflocher D, et al. Develop of a Quality Appraisal Tool for Case Series Studies Using a Modified Delphi Technique. Edmonton AB: Institue of Health Economics; 2012.

17. Higgins JPT, Thompson SG. Quantifying heterogeneity in a meta-analysis. Stat Med. 2002;21(11):1539-1558. doi:10.1002/sim.1186

18. Higgins JPT, Green S. Cochrane Handbook for Systematic Reviews of Interventions. Chichester: John Wiley \& Sons; 2011.

19. Allgayer H, Dietrich CF, Rohde W, Koch GF, Tuschhoff T. Prospective comparison of short- and long-term effects of pelvic floor exercise/ biofeedback training in patients with fecal incontinence after surgery plus irradiation versus surgery alone for colorectal cancer: clinical, functional and endoscopic/endosonographic findings. Scand J Gastroenterol. 2005;40:1168-1175. doi:10.1080/00365520510023477

20. Cong JC, Zhang H, Chen CS, Liu EQ. Biofeedback therapy can improve the anal sphincter function in patients with intersphincteric resection for low rectal cancer. World Chin J Digestol. 2006;25:2566-2570. doi:10.11569/wcjd.v14.i25.2566

21. Du P, Zi SM, Weng ZY, Chen W, Chen Y, Cui L. Biofeedback therapy for fecal incontinence in patients with mid or low rectal cancer after restorative resection. Zhonghua Wei Chang Wai Ke Za Zhi. 2010;8:580-582.

22. Bartlett L, Sloots K, Nowak M, Ho YH. Biofeedback therapy for symptoms of bowel dysfunction following surgery for colorectal cancer. Tech Coloproctol. 2011;15:319-326. doi:10.1007/s10151-011-0713-5

23. Cong JC, Chen CS, Zhang H, Qiao L, Liu EQ. Partial longitudinal resection of the anorectum and sphincter for very low rectal adenocarcinoma: a surgical approach to avoid permanent colostomy. Colorectal Dis. 2011;14:697-704. doi:10.1111/j.1463-1318.2011.02686.x

24. Kim K, Yu C. Effectiveness of biofeedback therapy in treatment of anterior resection syndrome after rectal cancer surgery. Dis Colon Rectum. 2011;53:662.

25. Laforest A, Bretagnol F, Mouazan AS, Maggiori L, Ferron M, Panis Y. Functional disorders after rectal cancer resection: does a rehabilitation programme improve anal continence and quality of life? Colorect Dis. 2012;14:1231-1237. doi:10.1111/j.1463-1318.2012.02956.x

26. Lee KH, Kim JS, Kim JY. Efficacy of biofeedback therapy for objective improvement of pelvic function in low anterior resection syndrome. Ann Surg Treat Res. 2019;97:194-201. doi:10.4174/astr.2019.97.4.194

27. Kuo LJ, Lin YC, Lai CH, et al. Improvement of fecal incontinence and quality of life by electrical stimulation and biofeedback for patients with low rectal cancer after intersphincteric resection. Arch Phys Med Rehabil. 2015;96:1442-1447. doi:10.1016/j.apmr.2015.03.013

28. Kye BH, Kim HJ, Kim G, Yoo RN, Cho HM. The effect of biofeedback therapy on anorectal function after the reversal of temporary stoma when administered during the temporary stoma period in rectal cancer patients with sphincter-saving surgery: the interim report of a prospective randomized controlled trial. Medicine (Baltimore). 2016;95:e3611. doi:10.1097/MD.0000000000003611

29. Liang Z, Ding W, Chen W, Wang Z, Du P, Cui L. Therapeutic evaluation of biofeedback therapy in the treatment of anterior resection syndrome after sphincter-saving surgery for rectal cancer. Clin Colorectal Cancer. 2016;15:e101-7. doi:10.1016/j.clcc.2015.11.002

30. Zheng MCWu XD, Fu CF, Chen YL, Kong LH, Pan ZZ. Intervention effect of biofeedback combined with pelvic floor muscle exercise on low anterior resection syndrome in patients with low anus-preserving rectal cancer. Zhonghua Yi Xue Za Zhi. 2019;99:2337. doi:10.3760/cma.j. issn.0376-2491.2019.30.004

31. Heymen S, Jones KR, Ringel Y, Scarlett Y, Whitehead WE. Biofeedback treatment of fecal incontinence: a critical review. Current Status. $2001 ; 44: 728-736$

32. Lundby L, Duelund-Jakobsen J. Management of fecal incontinence after treatment for rectal cancer. Curr Opin Support Palliat Care. 2011;5:60-64. doi:10.1097/SPC.0b013e3283435dd4

33. Massa L. Pelvic floor physical therapy interventions for oncology patients. Top Geriatr Rehabil. 2011;27:206-214. doi:10.1097/ TGR.0b013e3182198fc0

34. Juul T, Ahlberg M, Biondo S, et al. International validation of the low anterior resection syndrome score. Ann Surg. 2014;259:728-734. doi:10.1097/SLA.0b013e31828fac0b

35. Sedgwick P, Greenwood N. Understanding the Hawthorne effect. BMJ. 2015;351:h4672. doi:10.1136/bmj.h4672 
36. Bliss DZ, Mellgren A, Whitehead WE, et al. Assessment and Conservative Management of Faecal Incontinence and Quality of Life in Adults. Paris: 5th International Consultation on Incontinence; 2013.

37. Bharucha AE, Lacy BE. Mechanisms, evaluation, and management of chronic constipation. Gastroenterology. 2020;158:1232-1249.e3. doi:10.1053/j.gastro.2019.12.034

38. Bo K, Herbert RD. There is not yet strong evidence that exercise regimens other than pelvic floor muscle training can reduce stress urinary incontinence in women: a systematic review. J Physiother. 2013;59:159-168. doi:10.1016/S1836-9553(13)70180-2

39. Craig P, Dieppe P, Macintyre S, et al. Developing and evaluating complex interventions: the new Medical Research Council guidance. Int $J$ Nurs Stud. 2013;50:587-592. doi:10.1016/j.ijnurstu.2012.09.010

Therapeutics and Clinical Risk Management

Dovepress

\section{Publish your work in this journal}

Therapeutics and Clinical Risk Management is an international, peer-reviewed journal of clinical therapeutics and risk management, focusing on concise rapid reporting of clinical studies in all therapeutic areas, outcomes, safety, and programs for the effective, safe, and sustained use of medicines. This journal is indexed on PubMed Central, CAS, EMBase, Scopus and the Elsevier Bibliographic databases. The manuscript management system is completely online and includes a very quick and fair peer-review system, which is all easy to use. Visit http://www. dovepress.com/testimonials.php to read real quotes from published authors.

Submit your manuscript here: https://www.dovepress.com/therapeutics-and-clinical-risk-management-journal 